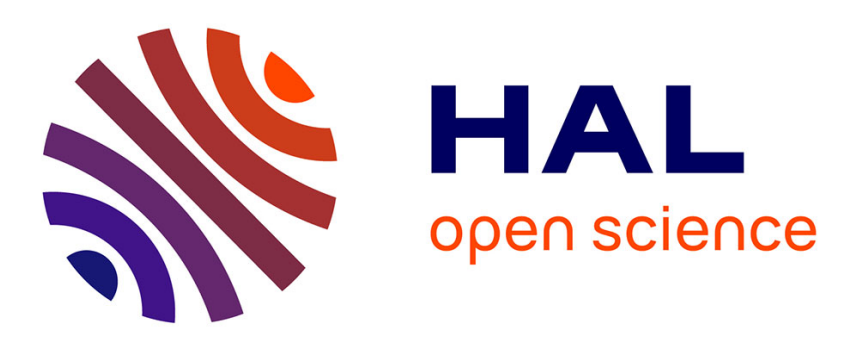

\title{
Effective flow surface of porous materials with two populations of voids under internal pressure: I. a GTN model
}

Pierre-Guy Vincent, Pierre Suquet, Yann Monerie, Hervé Moulinec

\section{- To cite this version:}

Pierre-Guy Vincent, Pierre Suquet, Yann Monerie, Hervé Moulinec. Effective flow surface of porous materials with two populations of voids under internal pressure: I. a GTN model. International Journal of Plasticity, 2014, 56, pp.45-73. 10.1016/j.ijplas.2013.11.013 . hal-00911873

\section{HAL Id: hal-00911873 \\ https://hal.science/hal-00911873}

Submitted on 30 Nov 2013

HAL is a multi-disciplinary open access archive for the deposit and dissemination of scientific research documents, whether they are published or not. The documents may come from teaching and research institutions in France or abroad, or from public or private research centers.
L'archive ouverte pluridisciplinaire HAL, est destinée au dépôt et à la diffusion de documents scientifiques de niveau recherche, publiés ou non, émanant des établissements d'enseignement et de recherche français ou étrangers, des laboratoires publics ou privés. 


\title{
Effective flow surface of porous materials with two populations of voids under internal pressure: I. a GTN model
}

\author{
Pierre-Guy Vincent ${ }^{\mathrm{a}, \mathrm{b}}$, Pierre Suquet ${ }^{\mathrm{c}, *}$, Yann Monerie ${ }^{\mathrm{a}, \mathrm{b}, 1}$, Hervé Moulinec $^{\mathrm{c}}$ \\ ${ }^{a}$ Institut de Radioprotection et de Sûreté Nucléaire, B.P. 3, 13115 Saint-Paul-lez-Durance Cedex, \\ France \\ ${ }^{b}$ Laboratoire de Micromécanique et d'Intégrité des Structures, IRSN-CNRS-UMII, B.P. 3, 13115 \\ Saint-Paul-lez-Durance Cedex, France \\ ${ }^{c}$ Laboratoire de Mécanique et d'Acoustique, CNRS, UPR 7051, Aix-Marseille Univ, Centrale \\ Marseille, 31, Chemin Joseph Aiguier, 13402 Marseille Cedex 20, France
}

\begin{abstract}
This study is devoted to the effective plastic flow surface of a bi-porous material saturated by a fluid. The material under consideration exhibits two populations of voids. The smaller voids are spherical voids whereas the larger ones are spheroidal and randomly oriented inside the material. These two populations of voids are subjected to internal pressures due to the presence of gases. Approximate models for the effective plastic flow surface of such a bi-porous saturated material have previously been proposed in (Vincent, P.-G., Monerie, Y., Suquet, P., 2009. Porous materials with two populations of voids under internal pressure: I. Instantaneous constitutive relations. International Journal of Solids and Structures 46, 480-506), where a three-scale homogenization procedure has been performed: first, smearing out all the small spherical bubbles using a Gurson-like matrix, and second, smearing out the intergranular ellipsoidal bubbles. Our objective here is to derive a simple analytical expression of the effective flow surface, starting from one of these previous models, obtained by generalizing the approach of (Gologanu, M., Leblond, J.B., Devaux, J., 1994. Approximate models for ductile metals containing non-spherical voids-case of axisymmetric oblate ellipsoidal cavities. ASME Journal of Engineering Materials and Technology 116, 290-297) to compressible materials. The main contributions of the present paper are: (1) an expression for the average dilatation-rate in the matrix, (2) an approximation of the effective flow surface in the form of a Gurson-Tvergaard-Needleman criterion. The accuracy of this new model is assessed in a companion paper by comparison with full field numerical simulations.
\end{abstract}

\footnotetext{
${ }^{*}$ Corresponding author. Tel: +334911642 08; fax: +33491164481

Email addresses: pierre-guy.vincent@irsn.fr (Pierre-Guy Vincent), suquet@lma.cnrs-mrs.fr (Pierre Suquet), yann.monerie@irsn.fr (Yann Monerie), moulinec@lma.cnrs-mrs.fr (Hervé Moulinec)

${ }^{1}$ Present address: Laboratoire de Mécanique et Génie Civil, Université Montpellier 2, CNRS, CC 048 Place Eugène Bataillon, 34095 Montpellier Cedex, France
} 
Keywords: A. Ductility, B. Constitutive behaviour, B. Ideally plastic material, B. Porous material, C. Variational calculus. 


\section{Motivations and objectives}

This study is devoted to the effective plastic flow surface of bi-porous materials characterized by the presence of two populations of voids with well separated sizes: (1) spherical intragranular bubbles at a very small scale, and (2) ellipsoidal (oblate) intergranular bubbles at a larger scale. These two populations of voids are filled with a fluid and therefore are subjected to internal pressures. They are randomly distributed inside the material with random orientation (for the ellipsoidal voids).

Such a microstructure is typical of the highly irradiated uranium dioxide $\left(\mathrm{UO}_{2}\right)$, a nuclear fuel commonly used in nuclear reactors. The assessment of the safety of nuclear plants under accident conditions has recently motivated several studies on the mechanical behavior of highly irradiated nuclear fuels at different scales (Vincent et al., 2008, 2009a,b), (Julien et al., 2011). $\mathrm{UO}_{2}$ is a polycrystalline material which exhibits, when highly irradiated, a very specific microstructure with two populations of voids of rather different sizes and shapes as shown in Figure 1 (more information about the formation and the size of these voids can be found in (Kashibe et al., 1993), (Lösönen, 2000), and (Kashibe and Une, 1991)):

- at the smallest scale (microscopic scale), a first population of voids, almost spherical in shape, is observed inside the grains. The diameter of these socalled intragranular voids is typically of the order of a few nanometers, much smaller than the typical grain size which is of the order of $10 \mu \mathrm{m}$ (Olander, 1976; Dherbey et al., 2002; Kashibe and Une, 1991).

- at a larger scale (mesoscopic scale), a second population of voids, roughly spheroidal in shape, is located at the grain boundaries (the diameter of these so-called intergranular voids is typically of the order of a few microns).

Both types of voids are filled with fission gases, with possibly different pressures inside the intergranular and intragranular voids. Under accident condition, the temperature in the material and the gas pressure inside the voids increases suddenly. At such high temperatures, this polycrystalline ceramic is ductile and the two populations of voids start growing until they eventually coalesce to form a macro-crack, a mechanism which is strongly reminiscent of ductile rupture in metals. The problem addressed in the present study is thus not restricted to highly irradiated $\mathrm{UO}_{2}$ and concerns more generally voided materials with two populations of pressurized voids.

The typical length scale attached to this polycrystalline material at the macroscopic scale is much larger than those of the two populations of voids leading to a clear separation of scales. Therefore the macroscopic properties of such bi-porous materials can be predicted by two successive homogenization procedures, a first one from the microscopic to the mesoscopic scale, and a second one from the mesoscopic to the macroscopic scale, as schematically illustrated in Figure 1b. 

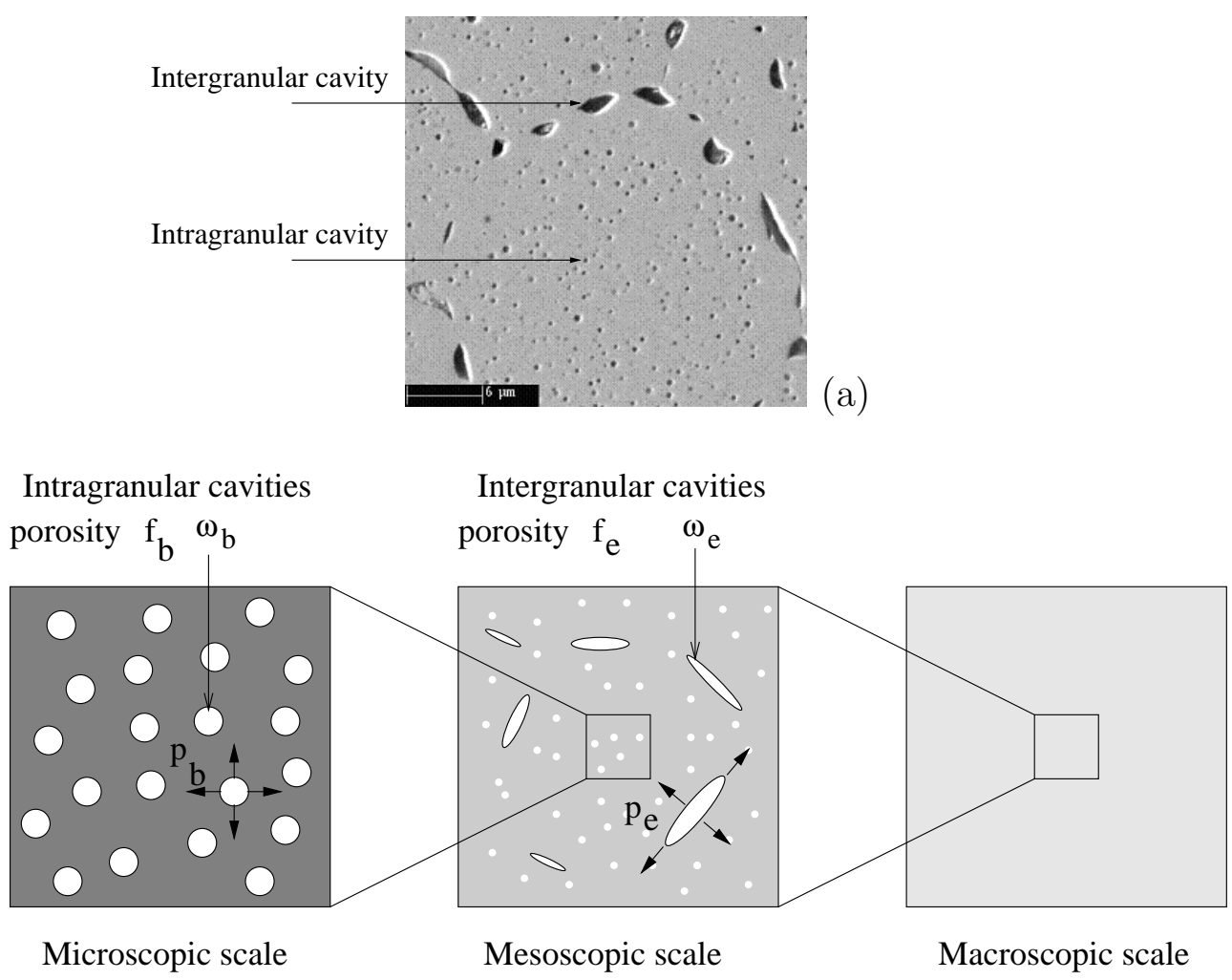

(b)

Figure 1: Micrography of irradiated $\mathrm{UO}_{2}(23 \mathrm{GW} d / \mathrm{tU})$ after heat treatment at $2000 \mathrm{~K}$ during $3 \mathrm{~h}$ (Dubourg et al., 2005). (b) Three-scale up-scaling (or homogenization) procedure (Vincent et al., 2009a).

The first scale transition, from the microscopic to the mesoscopic scale, will not be discussed here in much details. It amounts to finding the effective behavior of a porous material made from a von Mises matrix containing pressurized voids. Since the voids are spherical in shape with a rather low volume fraction Gurson's model (Gurson, 1977), with adequate modifications to account for the internal pressure in the voids, is a convenient and reasonably accurate model for the problem under consideration. More sophisticated homogenization models do exist, at least for viscoplasticity (Danas et al., 2008), but Gurson's model, in addition to its accuracy in the present context, has the definite advantage that it delivers an explicit form for the dissipation potential at the mesoscale which is needed for the second scale transition, from the mesoscopic to the macroscopic scale. This second step, to which most of this study is devoted, amounts to finding the effective properties of a voided material, composed of a Gurson matrix and containing randomly distributed ellipsoidal (spheroidal) voids. The distribution of the second population of voids assumed here is slightly different from that encountered in polycrystalline $\mathrm{UO}_{2}$, where the intergranular voids are located along the grain boundaries. This approximation is motivated by the results of Lebensohn et al. (2011) who showed that when the 
anisotropy of the single crystals is moderate (typically cubic materials such as $\mathrm{UO}_{2}$ ), the precise location of the intergranular voids is not very important. Another point worthy of notice is that a composite grain model could be constructed in the line of Vincent et al. (2009b) where the ellipsoidal voids are smeared out in a layer of material along the grain boundary surrounding a core of Gurson material. For the sake of simplicity and in order to obtain analytical results this model has not been pursued here.

The problem of a pressure-sensitive ductile matrix containing voids has been considered by different authors, Lee and Oung (1999), Guo et al. (2008) and Thoré et al. (2009) for a Drucker-Prager matrix containing spherical voids, Monchiet and Kondo (2012) for a Mises-Schleicher matrix, again with spherical voids. The problem of an incompressible matrix containing ellipsoidal voids in an incompressible matrix has been considered by Gologanu et al. (1994), Ponte Castañeda and Zaidman (1996), Benzerga et al. (2004), Monchiet et al. (2011), Madou and Leblond (2012) among others. The effect of a secondary population of small voids on the growth of a primary population of larger voids has been considered by several authors (Leblond and Perrin (1999), Fabrègue and Pardoen (2008) among others). But to the authors' knowledge the only work addressing specifically the problem of a Gurson matrix containing spheroidal voids under internal pressure is Vincent et al. (2009a) on which the present study is based.

Our objective here is to derive a simple analytical expression of the effective flow surface of such bi-porous materials under internal pressure building upon the previous study of Vincent et al. (2009a) where two rigorous upper bounds and an estimate of the effective flow surface have been derived. The first bound was based on the variational method of Ponte Castañeda (1991), also called the modified secant method (Suquet, 1995; Ponte Castañeda and Suquet, 1998) and was accurate at low stress-triaxiality. The second bound was obtained by generalizing the approach of Gurson (1977) and Gologanu et al. (1994) to compressible materials. The resulting prediction was observed to be accurate at high stress-triaxiality and this procedure is often referred to as the limit analysis procedure (LA). The estimate, called the $N$-phase model, inspired by the work of Bilger et al. (2002), was again based on the variational method of Ponte Castañeda (1991) and matches the best of the two bounds at low and high triaxialities. However, its implementation in a standard FEM code requires to solve, at each Gauss point of the structure, a set of $N$ nonlinear equations. This local nonlinear step results in a significant slowing-down of the structural calculation. The objective of the present work is therefore to derive an approximation in closed-form of the effective flow surface. This objective is motivated by the need of a model which can be accurate and at the same time cost effective for structural calculations. Such a model is also particularly useful for parametric studies to study the influence of the volume fraction and aspect ratio of the voids, as well as their internal pressure on the overall response of the material.

This objective is achieved by searching a "Gurson-like", or Gurson-Tvergaard- 
Needleman (GTN), approximation of the effective flow surface in the form:

$$
\frac{1}{\beta}\left(\frac{\Sigma_{e q}}{\sigma_{0}}\right)^{2}+\frac{1}{\alpha} \cosh \left(\frac{3}{2}\left(\frac{\Sigma_{m}}{\sigma_{0}}-\frac{\bar{\Sigma}_{m}^{-}+\bar{\Sigma}_{m}^{+}}{2 \sigma_{0}}\right)\right)-1=0,
$$

where $\Sigma_{m}$ is the macroscopic hydrostatic stress and $\Sigma_{e q}$ is the von Mises stress, $\Sigma_{m}=\frac{1}{3} \operatorname{Tr}(\boldsymbol{\Sigma}), \Sigma_{e q}=\sqrt{\frac{3}{2} \Sigma^{d}: \boldsymbol{\Sigma}^{d}}, \boldsymbol{\Sigma}^{d}=\boldsymbol{\Sigma}-\Sigma_{m} \boldsymbol{i}$ is the deviatoric part of $\boldsymbol{\Sigma}, \sigma_{0}$ denotes the yield stress of the unvoided matrix. $\bar{\Sigma}_{m}^{+}$and $\bar{\Sigma}_{m}^{-}$are the flow stresses of the material under hydrostatic stress (corresponding respectively to a contraction and a dilatation of the material), $\alpha$ and $\beta$ are the two scalar functions:

$$
\alpha=\cosh \left(\frac{3}{4} \frac{\bar{\Sigma}_{m}^{+}-\bar{\Sigma}_{m}^{-}}{\sigma_{0}}\right), \quad \beta=\left(\frac{\bar{\Sigma}_{e q}}{\sigma_{0}}\right)^{2} \frac{\alpha}{\alpha-1},
$$

where $\bar{\Sigma}_{e q}$ is the flow stress of the material under purely deviatoric strain-rate. $\alpha$, $\beta, \bar{\Sigma}_{m}^{+}$, and $\bar{\Sigma}_{m}^{-}$depend on the shape and volume fractions of the two populations of voids and on the internal pressures $p_{b}$ (in the intragranular voids) and $p_{e}$ (in the intergranular voids). Thus, the proposed criterion is a generalized Gurson criterion passing through the three specific points $\bar{\Sigma}_{m}^{+}, \bar{\Sigma}_{m}^{-}$, and $\bar{\Sigma}_{e q}$ as illustrated in Figure 2 .

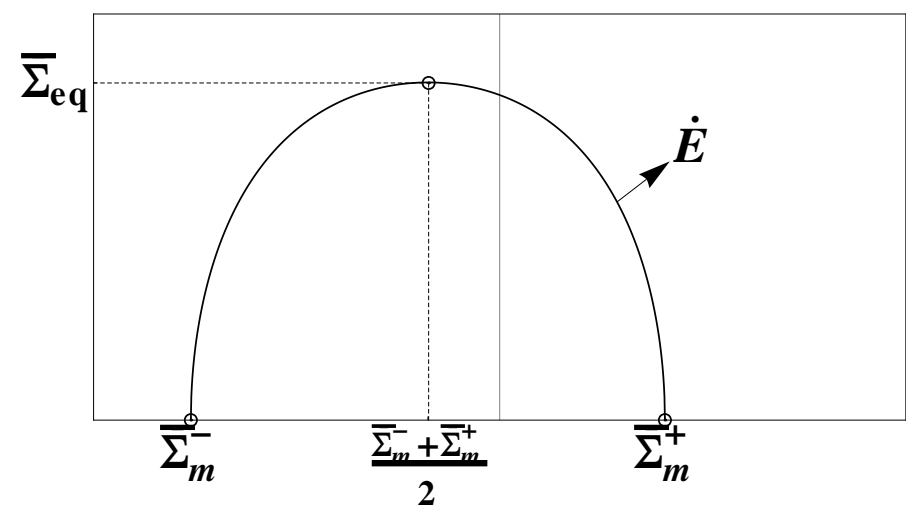

Figure 2: Generalized GTN criterion passing through the three specific points $\left(\bar{\Sigma}_{m}^{+}, 0\right),\left(\bar{\Sigma}_{m}^{-}, 0\right)$, $\left(\frac{\bar{\Sigma}_{m}^{-}+\bar{\Sigma}_{m}^{+}}{2}, \bar{\Sigma}_{e q}\right)$ corresponding respectively to purely hydrostatic (positive or negative) and deviatoric overall strain-rates.

As will be seen later, accurate approximations for these three specific points can be derived from the compressible velocity field of Vincent et al. (2009a) generalizing to compressible materials the approach of Gologanu et al. (1994). The key point in the determination of $\bar{\Sigma}_{m}^{+}, \bar{\Sigma}_{m}^{-}$, and $\bar{\Sigma}_{e q}$ is the estimation of the average dilatation-rate in the matrix, a new unknown by comparison with the more classical incompressible case. The analysis is performed on a hollow ellipsoid but the result remains valid for an assemblage of randomly oriented ellipsoids such as shown in Figure 3.

The present study, in two parts, is organized as follows. In the present paper, the different scales involved in the problem, the class of microstructures, the main 
assumptions of the model, the loading conditions, and classical relations used in upscaling methods are recalled in section 2 and the main results of Vincent et al. (2008, 2009a) are summarized. Then a simplified analytical model is derived in section 3 and 4 for spherical voids and in section 5 for spheroidal voids. The accuracy of this new model is assessed in part 2 of the paper by comparison with full field numerical simulations using the Fast Fourier Transform method of Moulinec and Suquet (1998), Michel et al. (2000), and Michel et al. (2001). These simulations are performed on microstructures containing a large number of spheroidal voids as shown in Figure 3 (b).

\section{Micromechanical analysis}

\subsection{Microstructure and loading conditions}

As exposed in section 1, the problem with primary and secondary populations of voids involves three different scales. The smallest (or microscopic) scale corresponds to the intragranular level (Figure 1b left). The intermediate (or mesoscopic) scale corresponds to the scale of a single grain or of a couple of grains with a large number of small intragranular voids (Figure 1b center). The largest (or macroscopic) scale corresponds to a large representative volume element containing a sufficiently large number of grains and intergranular voids together with an even larger number of intragranular voids.

Let $V$ denote a representative volume element at the macroscopic scale, $\omega_{e}$ is the domain occupied by the intergranular voids and $\omega_{b}$ is the domain occupied by the intragranular voids. The partial porosities $f_{b}, f_{e}$ and the total porosity $f$ read:

$$
f_{b}=\frac{\left|\omega_{b}\right|}{\left|V-\omega_{e}\right|}, \quad f_{e}=\frac{\left|\omega_{e}\right|}{|V|}, \quad f=f_{e}+f_{b}-f_{e} f_{b} .
$$

Since our main objective is to derive the instantaneous effective flow surface of the material (instantaneous in the terminology of Ponte Castañeda and Zaidman (1996) and Zaidman and Ponte Castañeda (1996)), the microstructure of the material is assumed to be fixed.

The spherical intragranular smaller voids are filled with fission gases at pressure $p_{b}$. The larger, intergranular, ellipsoidal voids are filled with fission gases at pressure $p_{e}$ which can be different from $p_{b}$. In addition, the whole volume element is subjected to an overall strain-rate $\dot{\boldsymbol{E}}$.

\subsection{Up-scaling}

The three scales of the problem being well separated, the up-scaling (or homogenization) procedure can be performed in two successive steps (see Figure 1(b)), first from the microscopic to the mesoscopic scale, smearing out all the small spherical voids, and second from the mesoscopic to the macroscopic scale, smearing out the details of the grain boundaries and the intergranular ellipsoidal voids. These two successive steps will be called the first scale transition and the second scale transition in the sequel. 


\subsubsection{Average stress and strain rate in voided materials}

The overall stress and strain-rate are the volume averages of the local stress and strain-rate fields over a representative volume element $V$ comprising a matrix domain and a domain occupied by voids denoted by $\omega$ (which can be $\omega_{b}$ or $\omega_{e}$, depending on the scale transition under consideration). The voids are subjected to a uniform pressure $p$ (which could either be $p_{b}$ or $p_{e}$ depending on the scale under consideration). The overall stress and strain rate are defined as in (Vincent et al., 2009a):

$$
\begin{gathered}
\boldsymbol{\Sigma}=\langle\boldsymbol{\sigma}\rangle=\frac{1}{|V|} \int_{V-\omega} \boldsymbol{\sigma} d \boldsymbol{x}-\frac{|\omega|}{|V|} p \boldsymbol{i}, \\
\dot{\boldsymbol{E}}=\langle\dot{\boldsymbol{\varepsilon}}\rangle=\frac{1}{|V|}\left(\int_{V-\omega} \dot{\boldsymbol{\varepsilon}} d \boldsymbol{x}-\int_{\partial \omega} \dot{\boldsymbol{u}} \otimes_{s} \boldsymbol{n} d s\right),
\end{gathered}
$$

where $\boldsymbol{\sigma}$ and $\dot{\boldsymbol{\varepsilon}}$ are the local stress and strain-rate fields, $\boldsymbol{n}$ is the unit normal vector on the boundary of the voids pointing from the bulk material towards the interior of the void, $\boldsymbol{i}$ is the second-order identity tensor, and $\boldsymbol{u}$ is the local velocity field. The symmetric tensor product of $\boldsymbol{a} \otimes_{s} \boldsymbol{b}$ of the two vectors $\boldsymbol{a}$ and $\boldsymbol{b}$ is defined as $\left(a \otimes_{s} b\right)_{i j}=\frac{1}{2}\left(a_{i} b_{j}+a_{j} b_{i}\right)$.

\subsubsection{Effective dissipation potential and effective flow surface}

The up-scaling procedure for the two scale transitions is based on the minimization of the average plastic dissipation in a representative volume element under an imposed macroscopic strain-rate to which corresponds a variational principle. The scale transition is not specified and the variational principle applies to both changes of scale:

$$
\boldsymbol{\Sigma}=\frac{\partial \Phi}{\partial \dot{\boldsymbol{E}}}(\dot{\boldsymbol{E}}, p),
$$

with

$$
\Phi(\dot{\boldsymbol{E}}, p)=\inf _{\boldsymbol{u} \in \mathcal{K}(\dot{\boldsymbol{E}})} \frac{1}{|V|}\left[\int_{V-\omega} \varphi(\varepsilon(\dot{\boldsymbol{u}})) d \boldsymbol{x}+p \int_{\partial \omega} \dot{\boldsymbol{u}} \cdot \boldsymbol{n} d s\right],
$$

where $\mathcal{K}(\dot{\boldsymbol{E}})$ denotes the set of velocity fields $\dot{\boldsymbol{u}}$ satisfying $\dot{\boldsymbol{u}}=\dot{\boldsymbol{E}} \boldsymbol{. x}$ on $\partial V$. The dissipation potential of the matrix, $\varphi$, is a positively homogeneous function of degree 1 with respect to $\dot{\varepsilon}$. $\Phi$ inherits this property of homogeneity of degree 1 , and therefore is not everywhere differentiable. Relation (6) must be understood in a generalized sense. $\Phi$ is the dissipation potential of an effective domain $P^{\text {hom }}(p)$ characterized as:

$$
P^{\text {hom }}(p)=\{\boldsymbol{\Sigma} \text { such that } \boldsymbol{\Sigma}: \dot{\boldsymbol{E}} \leq \Phi(\dot{\boldsymbol{E}}, p) \text { for all } \dot{\boldsymbol{E}}\} .
$$

The boundary of $P^{\text {hom }}(p)$ is the effective flow surface of the material and consists of all stresses deriving from the potential $\Phi$ :

$$
\text { when } \dot{\boldsymbol{E}} \neq \mathbf{0}, \boldsymbol{\Sigma}=\frac{\partial \Phi}{\partial \dot{\boldsymbol{E}}}(\dot{\boldsymbol{E}}, p) \text { belongs to the effective flow surface. }
$$

The variational principle (7) and the characterization (9) apply to both scale transitions. 


\subsubsection{First scale transition: generalized Gurson criterion}

For the first scale transition, the plastic dissipation potential $\varphi$ corresponds to a von Mises, incompressible, material $\left(\varphi(\dot{\boldsymbol{\varepsilon}})=\sigma_{0} \dot{\varepsilon}_{e q}\right)$. Generalizing Gurson's approach to account for the internal pressure in the voids, Vincent et al. (2009a) have established that the effective (mesoscopic) potential differs from the usual Gurson's potential by only a linear term

$$
\Phi^{b}\left(\dot{\boldsymbol{E}}, p_{b}\right)=\Phi^{\mathrm{Gur}}(\dot{\boldsymbol{E}})-3 p_{b} \dot{E}_{m}
$$

where Gurson's potential corresponding to the drained case $\left(p_{b}=0\right)$, reads:

$$
\Phi^{\mathrm{Gur}}(\dot{\boldsymbol{E}})=\sigma_{0} \int_{f_{b}^{*}}^{1}\left(\frac{4}{y^{2}}\left(\dot{E}_{m}\right)^{2}+\frac{1}{q_{3}}\left(\dot{E}_{e q}\right)^{2}\right)^{\frac{1}{2}} d y .
$$

$\dot{E}_{m}$ is the hydrostatic strain rate and $\dot{E}_{e q}$ is the von Mises norm for the strain rate, $\dot{E}_{m}=\frac{1}{3} \operatorname{Tr}(\dot{\boldsymbol{E}}), \dot{E}_{e q}=\sqrt{\frac{2}{3} \dot{\boldsymbol{E}}^{d}: \dot{\boldsymbol{E}}^{d}}, \dot{\boldsymbol{E}}^{d}=\dot{\boldsymbol{E}}-\dot{E}_{m} \boldsymbol{i}$ is the deviatoric part of $\dot{\boldsymbol{E}}$. The initial analysis of Gurson corresponds to $f_{b}^{*}=f_{b}$ and $q_{3}=1$. The enhanced porosity $f_{b}^{*}=q_{1} f_{b}$ and the parameter $q_{3}$ have been introduced by Koplik and Needleman (1988) to better match their unit-cell calculations. Detailed expressions for these parameters $q_{1}$ and $q_{3}$ can be found can be found in Koplik and Needleman (1988) and Leblond et al. (1994).

The effective flow surface corresponding to (10) through relation (9) is a generalized Gurson-Tvergaard-Needleman (GTN) criterion (Gurson, 1977; Koplik and Needleman, 1988; Tvergaard, 1990) :

$$
q_{3}\left(\frac{\Sigma_{e q}}{\sigma_{0}}\right)^{2}+2 q_{1} f_{b} \cosh \left(\frac{3}{2} \frac{\Sigma_{m}+p_{b}}{\sigma_{0}}\right)-1-\left(q_{1} f_{b}\right)^{2}=0,
$$

where $\Sigma_{m}$ is the hydrostatic stress and $\Sigma_{e q}$ is the von Mises stress, $\Sigma_{m}=\frac{1}{3} \operatorname{Tr}(\boldsymbol{\Sigma})$, $\Sigma_{e q}=\sqrt{\frac{3}{2} \Sigma^{d}: \Sigma^{d}}, \Sigma^{d}$ is the macroscopic stress deviator. Note that when the voids are subjected to a uniform internal pressure $p_{b}$, the general form of the Gurson criterion is preserved, $\boldsymbol{\Sigma}$ being replaced by $\boldsymbol{\Sigma}+p_{b} \boldsymbol{i}$.

\subsection{Second scale transition: the upper bound of Vincent et al. (2009a)}

For the second scale transition, the local dissipation potential $\varphi$ in (7) is the mesoscopic effective potential (10) corresponding to a compressible material, the voided domain consists of the intergranular voids $\omega=\omega_{e}$, the internal pressure is the pressure in the intergranular voids $p=p_{e}$ and the effective potential derived from (7) is the effective macroscopic potential $\Phi\left(\dot{\boldsymbol{E}}, p_{b}, p_{e}\right)$.

A more specific microstructure will be assumed at the mesoscopic scale, obtained by assembling confocal hollow ellipsoids in the line of Gologanu et al. (1994) 
or Madou and Leblond (2012) (who considered a single hollow ellipsoid). All intergranular voids are assumed to be spheroidal in shape with the same aspect-ratio $w=c / a$ between the semi-axis $c$ in the direction of the axis of the spheroid and the semi-axis $a$ in the plane orthogonal to this direction. The intergranular voids are therefore oblate when $w<1$, spherical when $w=1$ and prolate when $w>1$. Each void is surrounded by an ellipsoidal shell of matrix which is confocal to the void and the whole space is filled with such self-similar ellipsoids with arbitrary orientation and size (Figure 3 (b)). Vincent et al. (2009a) have derived rigorous properties and

(a)
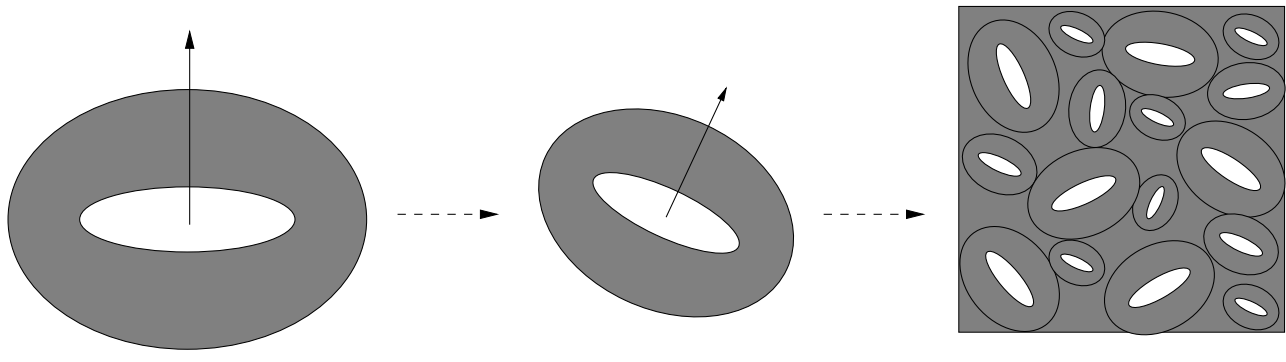

(b)

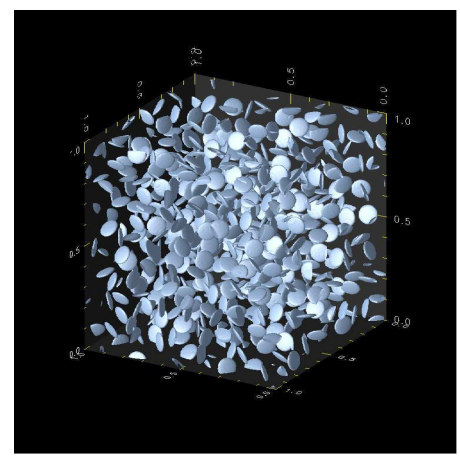

Figure 3: (a): Assemblage of self-similar randomly oriented hollow ellipsoids (from Vincent et al., 2009a). Unit pattern (left). Rotated and dilated hollow ellipsoid (center). Representative volume element (right). (b): Typical microstructure used in the full-field simulations: randomly oriented ellipsoidal voids (intergranular) in a compressible matrix (due to the presence of intragranular voids)

approximations for the effective potential $\Phi$. In particular they showed that

$$
\Phi\left(\dot{\boldsymbol{E}}, p_{b}, p_{e}\right)=\Phi\left(\dot{\boldsymbol{E}}, p_{b}-p_{e}, 0\right)-3 p_{e} \dot{E}_{m}=\Phi\left(\dot{\boldsymbol{E}}, 0, p_{e}-p_{b}\right)-3 p_{b} \dot{E}_{m} .
$$

In other words when the two populations of voids are saturated, with pressure $p_{b}$ in the small voids and $p_{e}$ in the large voids, and when the matrix is incompressible at the microscopic scale, it is sufficient to perform the second scale transition for a drained porous matrix $\left(p_{b}=0\right)$, provided that the pressure in the larger voids is set equal to $p_{e}-p_{b}$. Equivalently, it is sufficient to determine the potential $\Phi\left(\dot{\boldsymbol{E}}, p_{b}-p_{e}, 0\right)$ for a saturated porous matrix under pressure $p_{b}-p_{e}$, provided that the pressure in the larger voids is set equal to 0 . 
Moreover Vincent et al. (2009a) have derived a rigorous upper bound for the above described assemblage of hollow ellipsoids by generalizing to compressible materials the approach of Gologanu et al. (1994) by using for a single hollow ellipsoid the velocity field

$$
\dot{\boldsymbol{u}}=\dot{\boldsymbol{u}}^{G o l}+A \boldsymbol{x}
$$

where $A$ is a scalar parameter and $\dot{\boldsymbol{u}}^{\text {Gol }}$ is derived from the incompressible velocity field of Gologanu et al. (1994). A more detailed construction of the velocity field (14) is given in (Vincent et al., 2008) and (Vincent et al., 2009a). A definite advantage of this explicit field over the more general, but less explicit, family of velocity fields of Gologanu et al. (1997) is that it leads to an effective dissipation potential which can be expressed (almost) in closed form (see relation (40)). The matrix being isotropic (Gurson material) the use of enriched velocity fields is not expected to lead to significant improvements, although this point has not been checked in details. This velocity field can classically be extended to the hollow ellipsoid assemblage. The resulting strain-rate field in the matrix reads as

$$
\dot{\varepsilon}=\dot{\varepsilon}^{G o l}+A \boldsymbol{i},
$$

and since the field $\dot{\boldsymbol{\varepsilon}}^{G o l}$ is incompressible, $A$ can be interpreted as the dilatation rate in the matrix (which is assumed to be uniform by the specific choice (14)):

$$
\dot{\varepsilon}_{m}=A \text { in the matrix. }
$$

According to relation (5), the macroscopic dilatation-rate can be expressed as

$$
3 \dot{E}_{m}=3\left(1-f_{e}\right) A+f_{e} \frac{\left|\dot{\omega}_{e}\right|}{\left|\omega_{e}\right|}
$$

where $\left|\omega_{e}\right|$ denotes the volume of $\omega_{e}$. Keeping in mind that $f_{e}=\frac{\left|\omega_{e}\right|}{|V|}$, the change in volume fraction of the ellipsoidal voids can be expressed as

$$
\frac{\dot{f}_{e}}{f_{e}}=\frac{\left|\dot{\omega}_{e}\right|}{\left|\omega_{e}\right|}-\frac{|\dot{V}|}{|V|}=3\left(\dot{E}_{m}-A\right) \frac{1-f_{e}}{f_{e}} \quad \text { where } \quad \frac{|\dot{V}|}{|V|}=3 \dot{E}_{m} .
$$

In other words

- when $A=0$, there is no dilatation-rate in the matrix $\left(\dot{f}_{b}=3 f_{b} \dot{\varepsilon}_{m}=0\right)$ and the overall dilatation is entirely due to the change of volume of the intergranular voids,

- when $A=\dot{E}_{m}$, the volume fraction of the intergranular voids does not change $\left(\dot{f}_{e}=0\right)$ and the overall dilatation is entirely due to the change of volume of the intragranular voids. 
An accurate determination of the average dilatation rate $A$ in the matrix for arbitrary loading conditions $p_{b}, p_{e}$ and $\dot{\boldsymbol{E}}$ is essential in obtaining accurate predictions for the effective flow surface of the material. A numerical procedure was used in Vincent et al. (2009a). The present study aims at deriving an analytical approximation for this dilatation rate, using asymptotic expansions of $A$ as a function of $p_{b}-p_{e}$ and $\dot{\boldsymbol{E}}$ in the neighborhood of $A= \pm \infty, A=0$ and $A=\dot{E}_{m}$. Section 3 deals with the simpler case of spherical voids, whereas the general case of spheroidal and randomly oriented voids is addresses in section 5 .

\section{Spherical intergranular voids: average strain-rate in the matrix}

\subsection{The upper bound of Vincent et al. (2009a)}

When the intergranular voids are spherical $(w=1)$ (Vincent et al., 2009a) have shown that the effective potential $\Phi\left(\dot{\boldsymbol{E}}, p_{b}, p_{e}\right)$ for the composite sphere assemblage can be bounded from above by:

$$
\Phi^{+}\left(\dot{\boldsymbol{E}}, p_{b}, p_{e}\right)=\inf _{A} \phi(A, \dot{\boldsymbol{E}}, p)-3 p_{e} \dot{E}_{m}
$$

where $p=p_{b}-p_{e}$ and

$$
\phi(A, \dot{\boldsymbol{E}}, p)=\sigma_{0} \int_{f_{e}}^{1} \int_{f_{b}^{*}}^{1} \sqrt{4 \frac{A^{2}}{y^{2}}+4 \frac{\left(\dot{E}_{m}-A\right)^{2}}{q_{3} z^{2}}+\frac{\left(\dot{E}_{e q}\right)^{2}}{q_{3}}} d y d z-3\left(1-f_{e}\right) p A
$$

The effective flow surface corresponding to the potential (16) is defined by the parametric equations:

$$
\begin{aligned}
& \Sigma_{m}=\frac{1}{3} \frac{\partial \Phi^{+}}{\partial \dot{E}_{m}}\left(\dot{\boldsymbol{E}}, p_{b}, p_{e}\right)=-p_{e}+\frac{4}{3} \frac{\sigma_{0}}{q_{3}} \int_{f_{b}^{*}}^{1} \int_{f_{e}}^{1} \frac{\dot{E}_{m}-A_{\mathrm{sol}}}{z^{2} \sqrt{4 \frac{A_{\mathrm{sol}}^{2}}{y^{2}}+4 \frac{\left(\dot{E}_{m}-A_{\mathrm{sol}}\right)^{2}}{q_{3} z^{2}}+\frac{\left(\dot{E}_{e q}\right)^{2}}{q_{3}}}} d z d y \\
& \Sigma_{e q}=\frac{\partial \Phi^{+}}{\partial \dot{E}_{e q}}\left(\dot{\boldsymbol{E}}, p_{b}, p_{e}\right)=\frac{\sigma_{0}}{q_{3}} \int_{f_{b}^{*}}^{1} \int_{f_{e}}^{1} \frac{\dot{E}_{e q}}{\sqrt{4 \frac{A_{\mathrm{sol}}^{2}}{y^{2}}+4 \frac{\left(\dot{E}_{m}-A_{\mathrm{sol}}\right)^{2}}{q_{3} z^{2}}+\frac{\left(\dot{E}_{e q}\right)^{2}}{q_{3}}}} d z d y
\end{aligned}
$$

where $A_{\text {sol }}$ denotes the solution of the minimization problem (16). In a structural calculation the minimization problem (16) and the numerical integration of the double integrals in (18) have to be performed at every Gauss point of the structure. Both operations are time-consuming at a single material point and the cost at the level of a structure is extremely high. The rest of the paper aims first at determining an approximate, but accurate, analytical expression for the average dilatation-rate in the matrix $A_{\text {sol }}$ and second, to use this approximation to propose a simple analytical expression for the effective flow surface. 


\subsection{Specific properties of $A_{\text {sol }}$}

The approximation proposed is based on several general properties of $A_{\text {sol }}$ as a function of $p_{b}, p_{e}$ and $\dot{\boldsymbol{E}}$. Note that $A_{\text {sol }}$ depends in fact on $p_{b}$ and $p_{e}$ only through their difference $p=p_{b}-p_{e}$. The properties are listed below and proved in Appendix A.

Property 1: $A_{\text {sol }}(p, \dot{\boldsymbol{E}})$ is an odd function of $(p, \dot{\boldsymbol{E}})$ :

$$
A_{\text {sol }}(-p,-\dot{\boldsymbol{E}})=-A_{\text {sol }}(p, \dot{\boldsymbol{E}})
$$

A straightforward consequence of property 1 is that $\Phi^{+}$is itself an even function of $\left(\dot{\boldsymbol{E}}, p_{b}, p_{e}\right)$ :

$$
\Phi^{+}\left(-\dot{\boldsymbol{E}},-p_{b},-p_{e}\right)=\Phi^{+}\left(\dot{\boldsymbol{E}}, p_{b}, p_{e}\right) .
$$

Property 2: $A_{\text {sol }}(p, \dot{\boldsymbol{E}})$ is a monotone increasing function of $p$.

This property allows to consider either $A_{\text {sol }}$ as a function of $p$ (the determination of this function is our final goal), or conversely $p$ as a function of $A_{\text {sol }}$ (it will be useful to compute $p$ for a few, physically relevant, values of $A_{\text {sol }}$ ).

Property 3:

$$
\lim _{p \rightarrow \pm p^{\infty}} A_{\mathrm{sol}}(p, \dot{\boldsymbol{E}})= \pm \infty \quad \text { where } \quad p^{\infty}=\frac{2}{3} \frac{\sigma_{0}}{1-f_{e}} I_{0},
$$

where $I_{0}$ is a scalar function of $f_{e}$ and $f_{b}^{*}$ given by (A.7), and more specifically

$$
A_{\mathrm{sol}}(p, \dot{\boldsymbol{E}}) \sim \pm \sqrt{\frac{\sigma_{0}}{12 q_{3}\left(1-f_{e}\right)}} \sqrt{4 \dot{E}_{m}^{2} I_{1}+\dot{E}_{e q}^{2} I_{2}} \frac{1}{\sqrt{p^{\infty} \mp p}} \text { when } p \rightarrow \pm p^{\infty},
$$

where $I_{1}$ and $I_{2}$ are two scalar functions of $f_{e}$ and $f_{b}^{*}$ given by (A.10).

In other words the range of pressure $p$ for which the problem (16) has a solution is limited to the interval $]-p^{\infty},+p^{\infty}\left[\right.$. Explicit expressions of $I_{0}, I_{1}$, and $I_{2}$ are given in Appendix C.

Two other specific values of $p$, are worth investigating. The first one, $p_{0}$, corresponds to the pressure which compensates exactly the effect of the overall strain-rate $\dot{\boldsymbol{E}}$ and gives no average dilatation-rate in the matrix: $A_{\text {sol }}=0$ when $p=p^{0}$.

Property 4: $A_{\text {sol }}(p, \dot{\boldsymbol{E}})=0$ when $p=p^{0}(\dot{\boldsymbol{E}})$ with

$$
p^{0}(\dot{\boldsymbol{E}})=\frac{-4 \sigma_{0}\left(1-f_{b}^{*}\right)}{3 \sqrt{q_{3}}\left(1-f_{e}\right)} \dot{E}_{m} \int_{f_{e}}^{1} \frac{1}{z^{2}}\left(4 \frac{\dot{E}_{m}^{2}}{z^{2}}+\dot{E}_{e q}^{2}\right)^{-1 / 2} d z .
$$

In addition

$$
\frac{\partial A_{\text {sol }}}{\partial p}\left(p^{0}, \dot{\boldsymbol{E}}\right)=\frac{3\left(1-f_{e}\right)}{4 \sigma_{0} \sqrt{q_{3}}\left(1-f_{b}^{*}\right)} \frac{1}{\left[\frac{1}{f_{b}^{*}}\left(4 \dot{E}_{m}^{2} J_{1}+\dot{E}_{e q}^{2} J_{2}\right)+\frac{\dot{E}_{e q}^{2}}{q_{3}} J_{1}\right]},
$$


where $J_{1}$ and $J_{2}$ are functions of $f_{b}^{*}, f_{e}$ and $\dot{\boldsymbol{E}}$ defined in (A.13).

The second pressure of interest, $p_{1}$ corresponds to the internal pressure in the voids for which $A=\dot{E}_{m}$, which, following (15), corresponds to the specific loading conditions where the void volume fraction of the interganular voids is kept constant in time.

Property 5: $A_{\text {sol }}(p, \dot{\boldsymbol{E}})=\dot{E}_{m}$ when $p=p^{1}(\dot{\boldsymbol{E}})$ with

$$
p^{1}(\dot{\boldsymbol{E}})=\frac{4 \sigma_{0}}{3} \dot{E}_{m} \int_{f_{b}^{*}}^{1} \frac{1}{y^{2}}\left(4 \frac{\dot{E}_{m}^{2}}{y^{2}}+\frac{\dot{E}_{e q}^{2}}{q_{3}}\right)^{-1 / 2} d y .
$$

In addition

$$
\frac{\partial A_{\text {sol }}}{\partial p}\left(p^{1}, \dot{\boldsymbol{E}}\right)=\frac{3 q_{3}}{4 \sigma_{0}} \frac{1}{\left(\frac{1}{f_{e}} J_{3}+\dot{E}_{e q}^{2} J_{4}\right)},
$$

where $J_{3}$ and $J_{4}$ are functions of $f_{b}^{*}, f_{e}$ and $\dot{\boldsymbol{E}}$ defined in (A.17).

\subsection{Explicit approximation for $A_{\text {sol }}$}

An interpolation, matching the above exact asymptotic results when $p \sim-p^{\infty}$, $p \sim p^{0} p \sim p^{1}$, and $p \sim p^{\infty}$ is proposed.

Attention is restricted to $\dot{E}_{m}>0$, since according to (A.1), the expression of $A_{\text {sol }}$ for $\dot{E}_{m}<0$ can be deduced from that for $\dot{E}_{m}>0$ by changing the sign of $p$ and the sign of $A_{\mathrm{sol}}$.

The interval $]-p^{\infty},+p^{\infty}[$ is split into 3 subintervals and the proposed interpolation takes different forms in each of these intervals:

$$
\bar{A}(\dot{\boldsymbol{E}}, p)=\left\{\begin{array}{l}
A_{-}(\dot{\boldsymbol{E}}, p) \quad \text { when } \quad-p^{\infty}<p \leq p^{0} \\
A_{0}(\dot{\boldsymbol{E}}, p) \quad \text { when } \quad p^{0} \leq p \leq p^{1} \\
A_{+}(\dot{\boldsymbol{E}}, p) \quad \text { when } \quad p^{1} \leq p<p^{\infty}
\end{array}\right.
$$

The branches are chosen in such a way that the singularity of $\left.A_{\text {sol }}(p, \dot{\boldsymbol{E}})\right)$ at $p= \pm p^{\infty}$ is reproduced and the exact values of $\left.A_{\text {sol }}(p, \dot{\boldsymbol{E}})\right)$ and $\frac{\partial A_{\text {sol }}}{\partial p}(p, \dot{\boldsymbol{E}})$ are met at $p=p^{0}$ and $p=p^{1}$ (the dependence on $\dot{\boldsymbol{E}}$ is omitted for simplicity):

$$
\begin{gathered}
A_{-}\left(p^{0}\right)=A_{0}\left(p^{0}\right)=0, \quad \frac{\partial A_{-}}{\partial p}\left(p^{0}\right)=\frac{\partial A_{0}}{\partial p}\left(p^{0}\right)=\frac{\partial A_{\text {sol }}}{\partial p}\left(p^{0}\right) \\
A_{0}\left(p^{1}\right)=A_{+}\left(p^{1}\right)=\dot{E}_{m}, \quad \frac{\partial A_{0}}{\partial p}\left(p^{1}\right)=\frac{\partial A_{+}}{\partial p}\left(p^{1}\right)=\frac{\partial A_{\mathrm{sol}}}{\partial p}\left(p^{1}\right)
\end{gathered}
$$

In the first interval $]-p^{\infty}, p_{0}[$ the proposed approximation reads as:

$$
A_{-}(p)=-C_{-}\left(\frac{\sqrt{p^{\infty}+p^{0}}}{\sqrt{p^{\infty}+p}}-1\right)+D_{-}\left(p-p^{0}\right)
$$


with

$$
\left.\begin{array}{l}
C_{-}=\frac{1}{\sqrt{p^{\infty}+p^{0}}} \sqrt{\frac{\sigma_{0}}{12 q_{3}\left(1-f_{e}\right)}} \sqrt{4 \dot{E}_{m}^{2} I_{1}+E_{e q}^{2} I_{2}}, \\
D_{-}=\frac{\partial A_{\mathrm{sol}}}{\partial p}\left(p^{0}\right)-\frac{C_{-}}{2\left(p^{\infty}+p^{0}\right)} .
\end{array}\right\}
$$

Similarly, the interpolation proposed for the interval $] p_{1},+p^{\infty}[$ reads as:

$$
A_{+}(p)=C_{+}\left(\frac{\sqrt{p^{\infty}-p^{1}}}{\sqrt{p^{\infty}-p}}-1\right)+D_{+}\left(p-p^{1}\right)+\dot{E}_{m}
$$

with

$$
\left.\begin{array}{rl}
C_{+} & =\frac{1}{\sqrt{p^{\infty}-p^{1}}} \sqrt{\frac{\sigma_{0}}{12 q_{3}\left(1-f_{e}\right)}} \sqrt{4 \dot{E}_{m}^{2} I_{1}+E_{e q}^{2} I_{2}}, \\
D_{+} & =\frac{\partial A_{\mathrm{sol}}}{\partial p}\left(p^{1}\right)-\frac{C_{+}}{2\left(p^{\infty}-p^{1}\right)} .
\end{array}\right\}
$$

As for the middle interval $] p_{0}, p_{1}\left[\right.$, the function $A_{\text {sol }}(p)$ is approximated by a polynom of degree 3 in $p$ matching the exact values of $A_{\text {sol }}(p)$ and its first derivative at $p=p_{0}$ and $p=p_{1}$ :

$$
A_{0}(p)=\left(p-p^{0}\right)\left(\frac{E}{2}\left(p-p^{1}\right)^{2}+F\left(p-p^{1}\right)+\frac{\dot{E}_{m}}{p^{1}-p^{0}}\right)
$$

with

$$
\left.\begin{array}{l}
E=\frac{2}{\left(p^{1}-p^{0}\right)^{2}}\left(\frac{\partial A_{\mathrm{sol}}}{\partial p}\left(p^{0}\right)+\frac{\partial A_{\mathrm{sol}}}{\partial p}\left(p^{1}\right)-\frac{2 \dot{E}_{m}}{p^{1}-p^{0}}\right), \\
F=\frac{1}{p^{1}-p^{0}}\left(\frac{\partial A_{\mathrm{sol}}}{\partial p}\left(p^{1}\right)-\frac{\dot{E}_{m}}{p^{1}-p^{0}}\right)
\end{array}\right\}
$$

\subsection{Comparison between the exact minimizer $A_{\text {sol }}$ and the approximation $\bar{A}$ (27)}

The approximate form (27) is compared in this section with the exact minimizer $A_{\text {sol }}$ of (16). This is done for three different values of the triaxiality ratio of the overall strain-rate: $\dot{E}_{m} / \dot{E}_{e q}=0, \dot{E}_{m} / \dot{E}_{e q}=+\infty$ and $\dot{E}_{m} / \dot{E}_{e q}=1$.

\subsubsection{Purely deviatoric macroscopic strain-rate: $\dot{E}_{m}=0$.}

When the overall strain-rate is purely deviatoric, the two intermediate pressures $p^{0}$ and $p^{1}$ are both equal to 0 and the intermediate regime (between $p^{0}$ and $p^{1}$ ) no more exists. The approximation (27) takes a simplified form thanks to the expressions given in Appendix A.1. The exact minimizer of (16) (crosses) and the approximation (27) (solid line) are compared in Figure 4 for four different values of the volume fraction of both populations of voids. As can be seen, the agreement is excellent over the whole range of admissible pressures $p$, and for the four values of the porosities. 


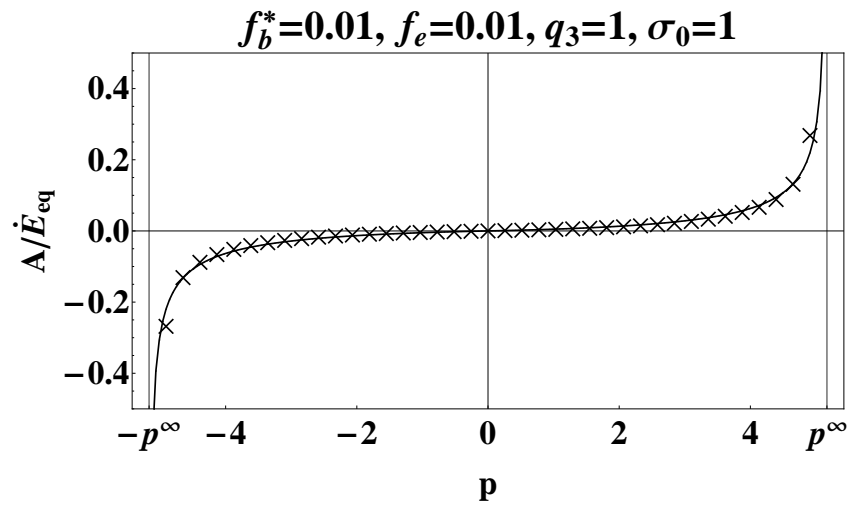

(a)

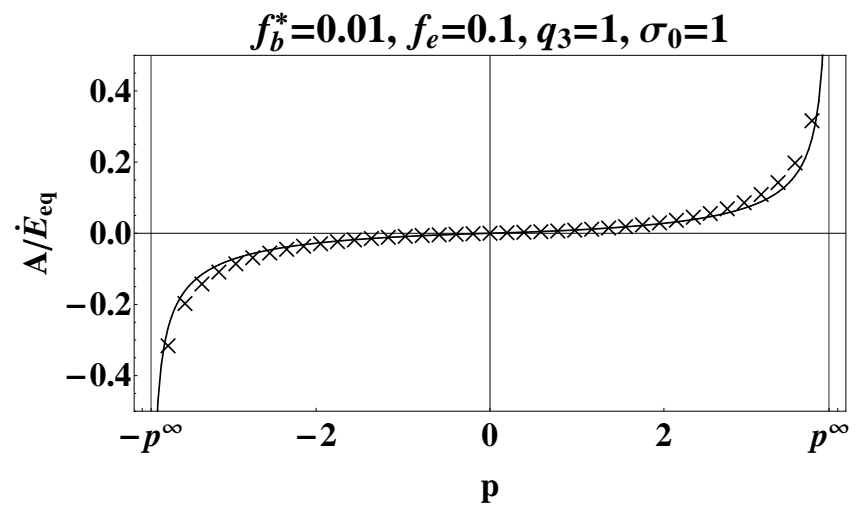

(c)

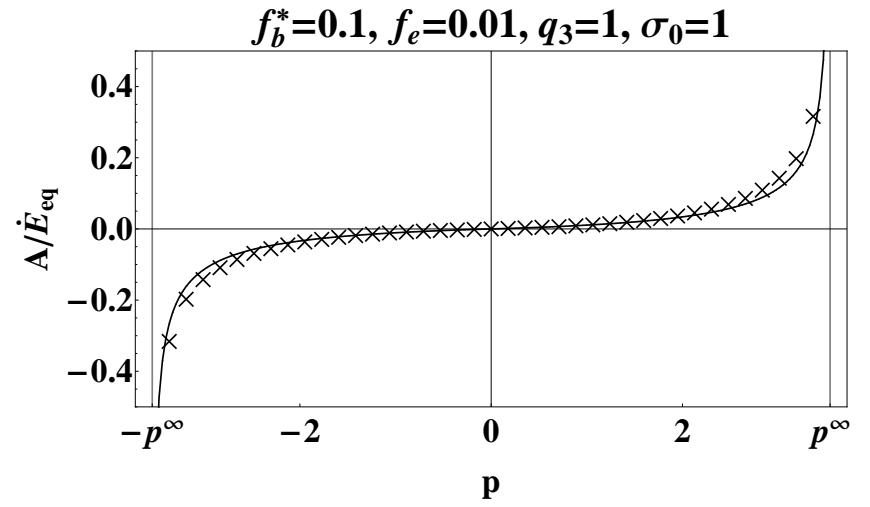

(b)

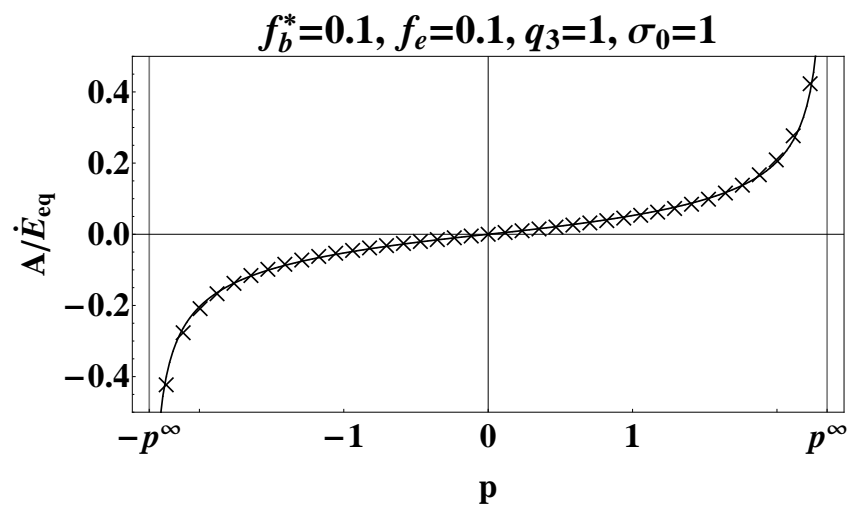

(d)

Figure 4: Spherical intergranular voids. Purely deviatoric overall strain-rate $\dot{E}_{m}=0$. Comparison between the exact dilatation-rate $A_{\text {sol }}$ (crosses) and the approximation $\bar{A}$ (27) (solid line). 


\subsubsection{Purely hydrostatic macroscopic strain-rate: $\dot{E}_{e q}=0$.}

When the overall strain-rate is purely hydrostatic, the two intermediate pressures $p^{0}$ and $p^{1}$ can be computed explicitly (see Appendix A.2). Other simplifications occur in the different terms of the approximation (27) which takes a simplified form thanks to the expressions given in Appendix A.2. Again, the agreement between the exact minimizer of (16) (crosses) and the approximation (27) (solid line) is excellent over the whole range of admissible pressures $p$, and for the four values of the porosities (Figure 5).

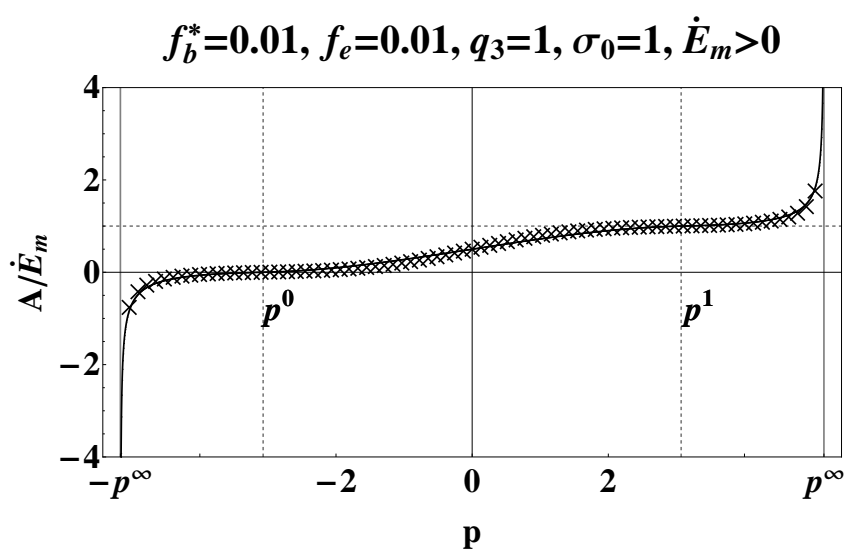

(a)

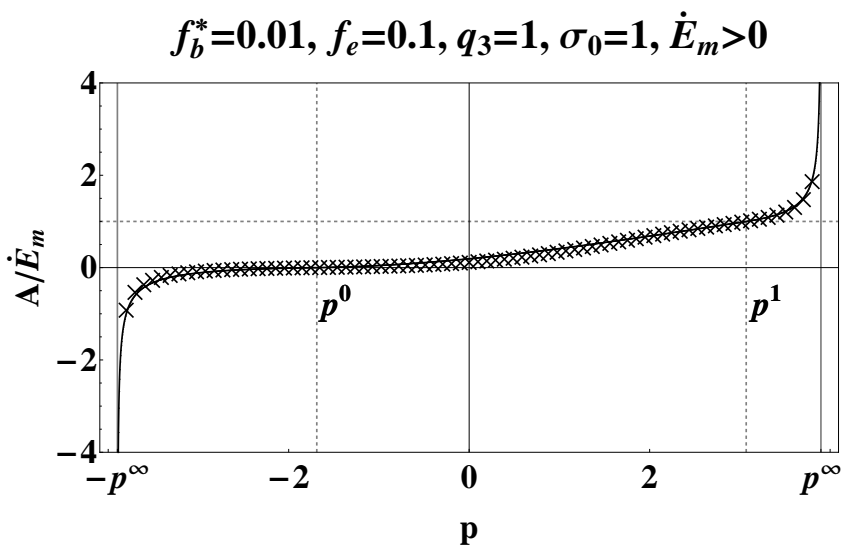

(c)

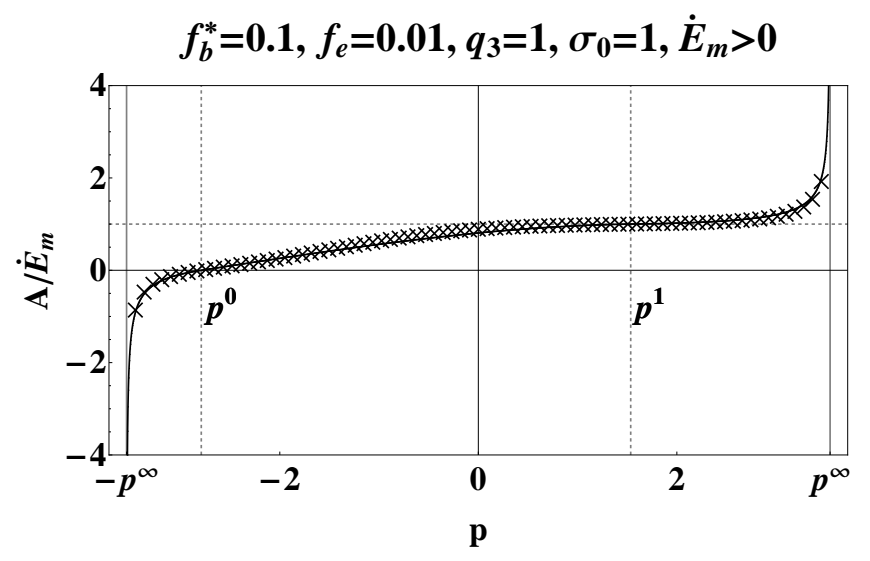

(b)

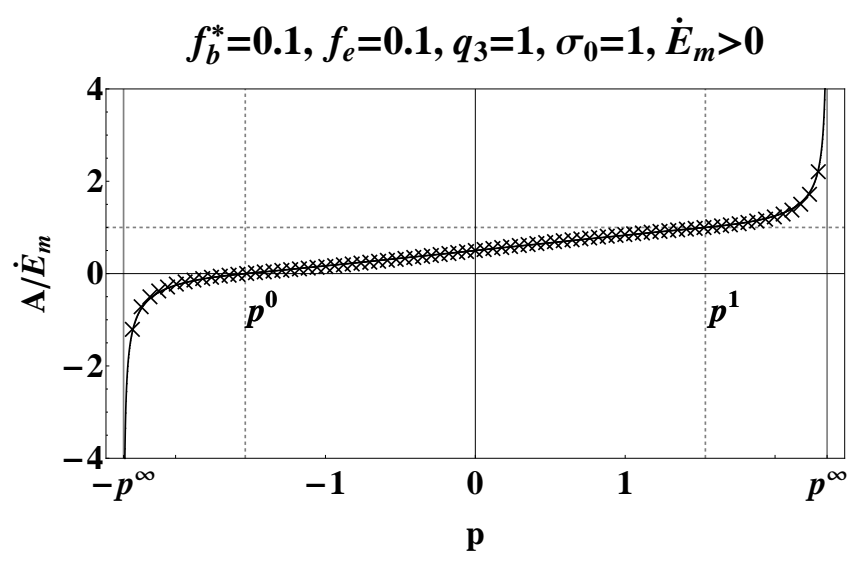

(d)

Figure 5: Spherical intergranular voids. Purely hydrostatic overall strain-rate $\dot{E}_{e q}=0$. Comparison between the exact dilatation-rate $A_{\text {sol }}$ (crosses) and the approximation $\bar{A}(27)$ (solid line).

3.4.3. Intermediate strain-rate: $\dot{E}_{m} / \dot{E}_{e q}=1$.

For all intermediate values of the triaxiality ratio of the overall strain-rate, the integrals have to be computed numerically. A specific triaxiality ratio $\dot{E}_{m} / \dot{E}_{e q}=1$ 
has been considered for the comparison performed in Figure 6. Again the agreement between the exact minimizer and its approximation is seen to be excellent.

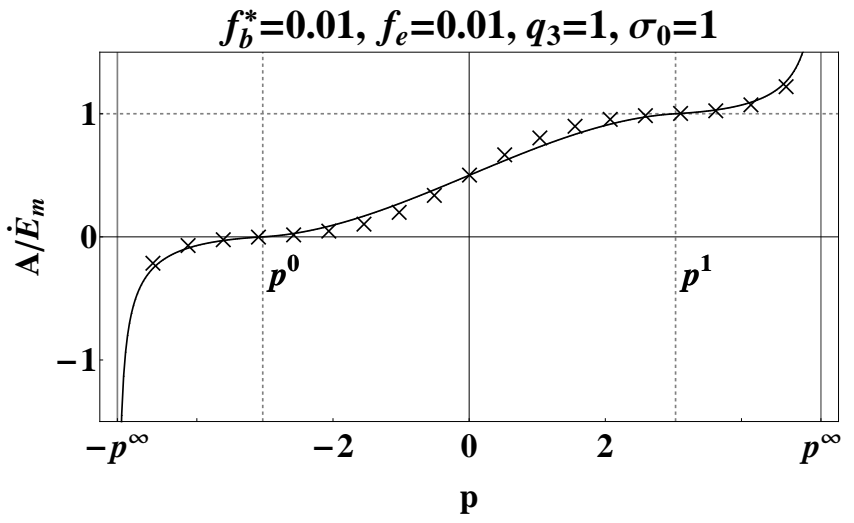

(a)

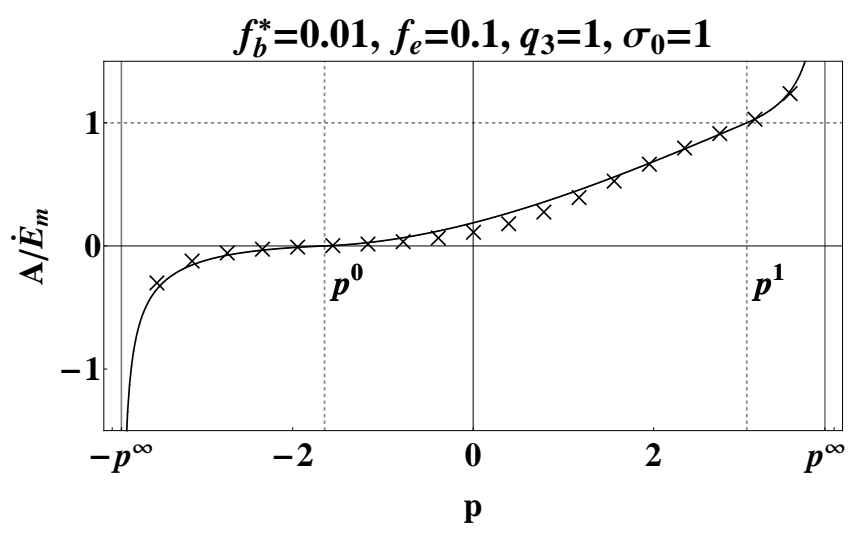

(c)

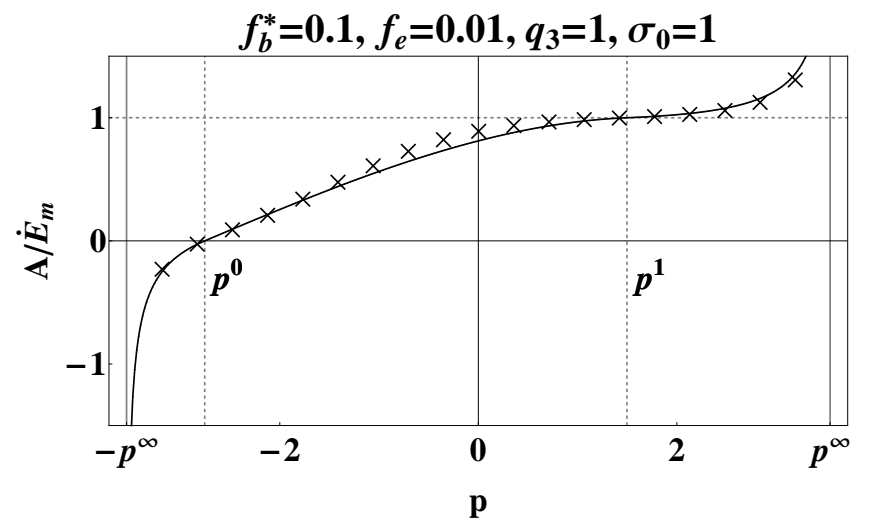

(b)

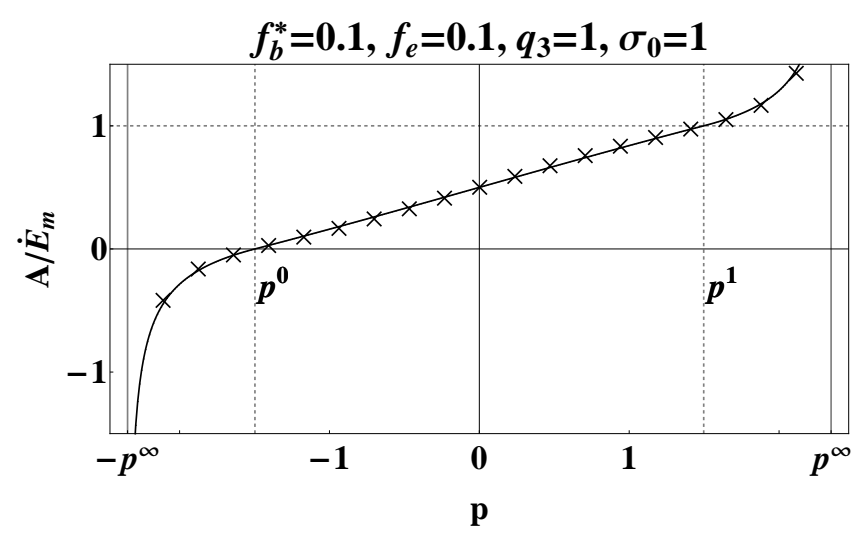

(d)

Figure 6: Spherical intergranular voids. Triaxial overall strain-rate: $\dot{E}_{m} / \dot{E}_{e q}=1$. Comparison between the exact dilatation-rate $A_{\text {sol }}$ (crosses) and the approximation $\bar{A}(27)$ (solid line).

All these comparisons show that $A_{\text {sol }}$, the average dilatation-rate in the matrix, solution of the variational problem (16) can be accurately approximated by the expression (27). Consequently, the resolution of the minimization problem (16) can be avoided.

\section{A GTN criterion for pressurized voided materials with spherical in- tergranular voids}

It remains to find an accurate approximation for the effective flow surface defined by the parametric equations (18). A first approach is to use the approximation $\bar{A}$ in (16) to get an upper bound, denoted by $\bar{\Phi}$, for the overall dissipation potential:

$$
\bar{\Phi}\left(\dot{\boldsymbol{E}}, p_{b}, p_{e}\right)=\phi(\bar{A}(p, \dot{\boldsymbol{E}}), \dot{\boldsymbol{E}}, p)-3 p_{e} \dot{E}_{m} .
$$


Then the approximate flow surface is defined by the parametric equations:

$$
\Sigma_{m}=\frac{1}{3} \frac{\partial \bar{\Phi}}{\partial \dot{E}_{m}}\left(\dot{\boldsymbol{E}}, p_{b}, p_{e}\right), \quad \Sigma_{e q}=\frac{\partial \bar{\Phi}}{\partial \dot{E}_{e q}}\left(\dot{\boldsymbol{E}}, p_{b}, p_{e}\right)
$$

It should be noted that the parametric equations (35) do not reduce to (18) upon substitution of $A_{\text {sol }}$ with $\bar{A}$. In addition to the double integrals in (18), other terms involving the derivatives of $\bar{A}$ with respect to $\dot{E}_{m}$ and $\dot{E}_{e q}$ have to be taken into account. The resulting flow surface is a rigorous outer bound to the flow surface defined by the potential $\Phi^{+}$. A comparison between the two flow surfaces is shown in Figure 7 where they are respectively plotted with crosses and squares. The agreement between crosses and squares is seen to be excellent which proves that the expression (27) is an accurate approximation of the actual solution $A_{\text {sol }}$.

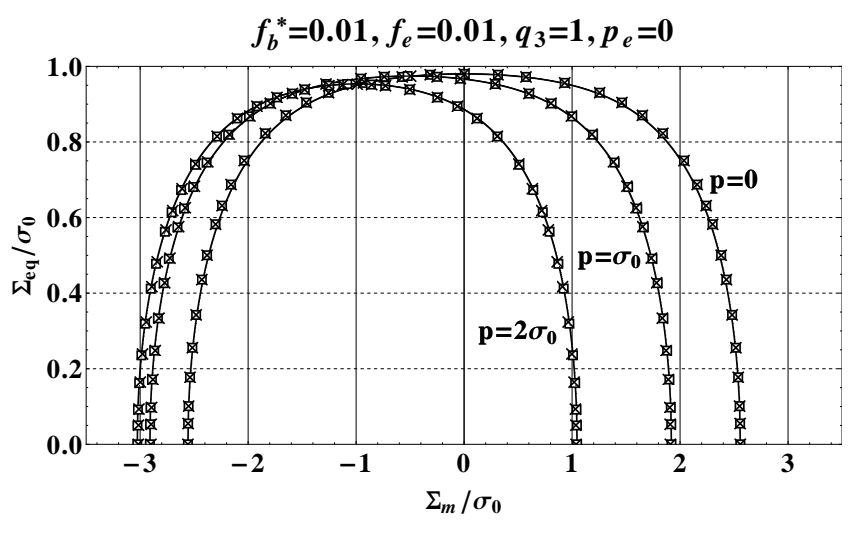

(a)

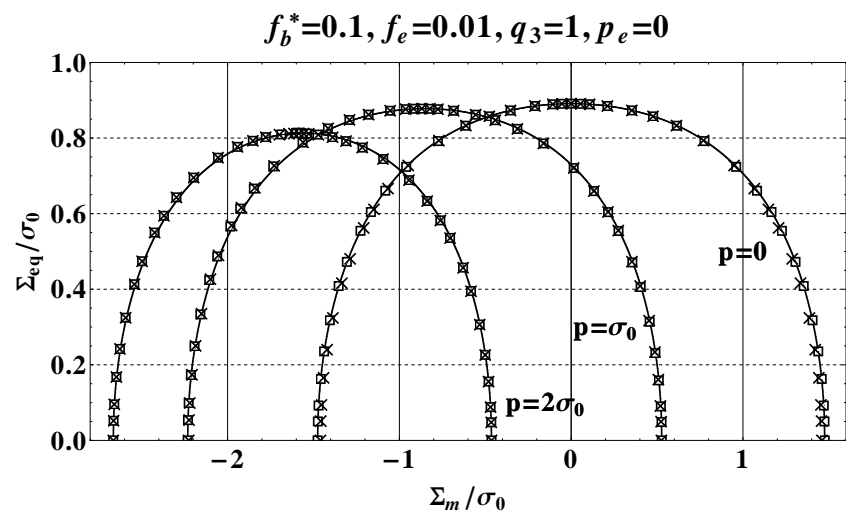

(c)

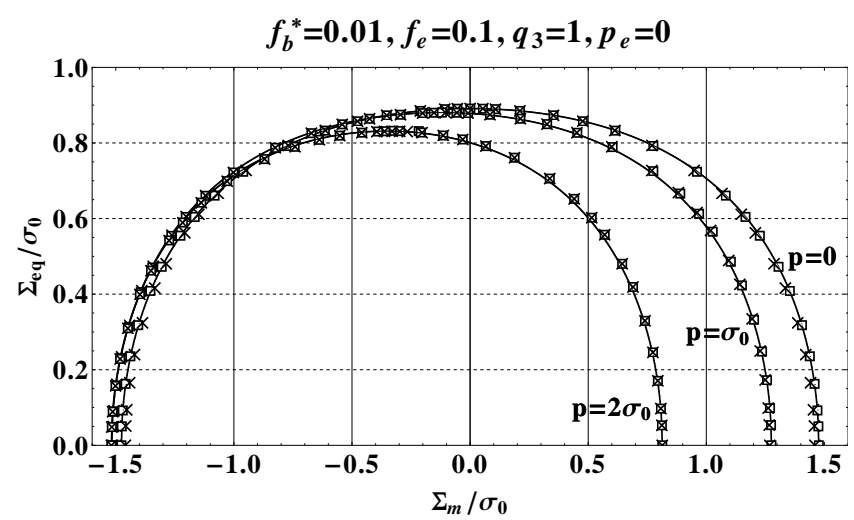

(b)

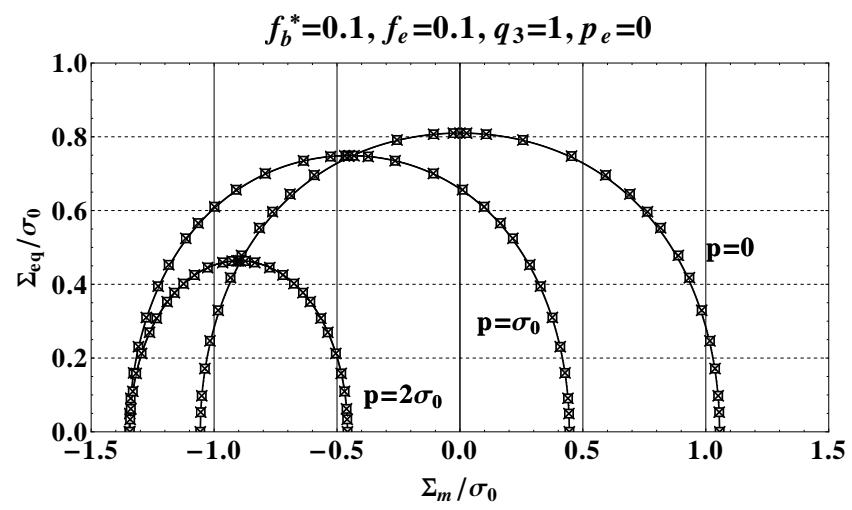

(d)

Figure 7: Spherical intergranular voids. Comparison between the flow surface (18) (crosses), the flow surface (35) (squares) and the GTN approximation (1) (solid line). $p$ denotes the difference $p_{b}-p_{e}$.

The cost for determining the flow surface (35) is certainly less than that for 
(18), as there is no minimization problem to be solved for $A_{\text {sol }}$. However the double integrals are still time-consuming (keeping in mind that this operation has to be repeated at every Gauss point of a piece of material, at every time step along its loading history) and a simpler analytical expression for this flow surface is searched for.

To this end, a useful observation can be made from Figure 7: the shape of the two flow surfaces (18) and (35) is reminiscent of a GTN flow surface whose equation is (1). As schematically depicted in Figure 2, such a GTN surface is completely determined by the data of three particular stress points: the two points $\left(\bar{\Sigma}_{m}^{-}, 0\right)$ and $\left(\bar{\Sigma}_{m}^{+}, 0\right)$ on the hydrostatic axis and the point $\left(\frac{1}{2}\left(\bar{\Sigma}_{m}^{-}+\bar{\Sigma}_{m}^{+}\right), \bar{\Sigma}_{e q}\right)$ corresponding to the maximum deviatoric stress on the surface. Obviously, looking for an approximation in the form (1) for the flow surface, leads to enforcing certain symmetries which are not necessarily present in the actual surface. This is discussed in Appendix D.

The two hydrostatic points $\left(\bar{\Sigma}_{m}^{ \pm}, 0\right)$ correspond to the two purely hydrostatic strain rates $\dot{\boldsymbol{E}}= \pm \boldsymbol{i}$ where $\boldsymbol{i}$ is the identity among second-order tensors. Indeed $\bar{A}$ and consequently $\bar{\Phi}$ depend on the two first invariants of $\dot{\boldsymbol{E}}$. Therefore, the constitutive relations obtained by derivation of the potential are isotropic and the principal axes of the overall stress and of the overall strain-rate coincide. Every direction is a principal direction for $\dot{\boldsymbol{E}}$ and consequently for $\boldsymbol{\Sigma}$ which proves that $\boldsymbol{\Sigma}$ is hydrostatic as well. The points $\left(\bar{\Sigma}_{m}^{ \pm}, 0\right)$ could be computed using the relations (35) with $\dot{\boldsymbol{E}}= \pm \boldsymbol{i}$. But it is simpler to use a specific property of positively homogeneous functions of degree 1 . Both $\Phi^{+}$and $\bar{\Phi}$ are such that

$$
\bar{\Phi}(\lambda \dot{\boldsymbol{E}})=\lambda \bar{\Phi}(\dot{\boldsymbol{E}}) \quad \text { for all positive } \lambda
$$

By derivation with respect to $\lambda$ (and setting $\lambda=1$ ), one gets (Euler's equation):

$$
\frac{\partial \bar{\Phi}}{\partial \dot{\boldsymbol{E}}}(\dot{\boldsymbol{E}}): \dot{\boldsymbol{E}}=\bar{\Phi}(\dot{\boldsymbol{E}})
$$

It follows from this relation applied with $\dot{\boldsymbol{E}}= \pm \boldsymbol{i}$ that

$$
3 \bar{\Sigma}_{m}^{ \pm}= \pm \phi\left(\bar{A}^{ \pm}, \pm \boldsymbol{i}, p\right)-3 p_{e}
$$

where $\phi$ is defined in (17) and $\bar{A}^{ \pm}=\bar{A}( \pm \boldsymbol{i}, p)$ where the function $\bar{A}$ is defined in (27). Similarly, choosing a purely deviatoric strain-rate $\dot{\boldsymbol{E}}^{d}$ with $\dot{E}_{e q}^{d}=1$ in (36) yields

$$
\bar{\Sigma}_{e q}=\phi\left(\bar{A}_{e q}, \dot{\boldsymbol{E}}^{d}, p\right)
$$

where $\bar{A}_{e q}=\bar{A}\left(\dot{\boldsymbol{E}}^{d}, p\right)$ with the function $\bar{A}$ defined in $(27)$. The quantities $\bar{A}^{ \pm}$ and $\bar{A}_{e q}$ take simplified forms thanks to the expressions given in Appendix A.1 and Appendix A.2. Then the flow surface corresponding to $\bar{\Phi}$ is bounded all the 
hydrostatic and the deviatoric axes by:

$$
\left.\begin{array}{l}
\bar{\Sigma}_{m}^{ \pm}= \pm \frac{\sigma_{0}}{3} \int_{f_{e}}^{1} \int_{f_{b}^{*}}^{1} \sqrt{4 \frac{\left(\bar{A}^{ \pm}\right)^{2}}{y^{2}}+4 \frac{\left( \pm 1-\bar{A}^{ \pm}\right)^{2}}{q_{3} z^{2}}} d y d z-\left(1-f_{e}\right) p \bar{A}^{ \pm}-p_{e} \\
\bar{\Sigma}_{e q}=\sigma_{0} \int_{f_{e}}^{1} \int_{f_{b}^{*}}^{1} \sqrt{4 \frac{\bar{A}_{e q}^{2}}{y^{2}}+4 \frac{\bar{A}_{e q}^{2}}{q_{3} z^{2}}+\frac{1}{q_{3}}} d y d z-3\left(1-f_{e}\right) p \bar{A}_{e q} .
\end{array}\right\}
$$

The integrals entering (39) can be expressed in closed form (see Appendix C).

4.1. Comparison between the GTN approximation and the flow surfaces associated with the upper bounds $\Phi^{+}$and $\bar{\Phi}$.

The three flow surfaces corresponding to the potential $\Phi^{+}(16)$ of Vincent et al. (2009a), the present potential $\bar{\Phi}$ and the approximation (1) are compared in Figure 7. As can be seen the agreement is very satisfactory at all stress triaxiality. A more accurate comparison, focusing on the three specific overall stresses corresponding respectively to the three overall strain-rate states $\dot{\boldsymbol{E}}= \pm \boldsymbol{i}$ and $\dot{\boldsymbol{E}}=\dot{\boldsymbol{E}}^{d}$, has been performed over a broad range of porosities. These stress states provide rigorous bounds for the effective flow surface on the hydrostatic axis and on the deviatoric axis respectively. These three stress states are successively evaluated with the actual solution $A_{\text {sol }}$ of the minimization problem (16) (the corresponding stresses are denoted by $\left.\Sigma_{m}^{\mathrm{sol} \pm}, \Sigma_{e q}^{\mathrm{sol}}\right)$ and with the approximation $\bar{A}$ (the corresponding stresses are denoted by $\bar{\Sigma}_{m}^{ \pm}, \Sigma_{e q}$ ). As can be seen in Figure 8 , the discrepancy between the two sets of results is less than $1.5 \%$ over the whole range of porosities $f_{b}^{*}$ and $f_{e}$ of interest in the present context of nuclear fuel.

\section{Ellipsoidal intergranular voids}

\subsection{Upper bound and approximation of Vincent et al. (2009a)}

The present section is devoted to ellipsoidal voids, and more specifically to spheroidal voids characterized by two equal axes. In view of Figure 1a, attention is restricted to oblate spheroids where the third axis is smaller than the two equal axes (see Appendix E for more details). The voids are randomly distributed and randomly oriented in the volume element $V$ and the matrix surrounding them is a Gurson material. The additional porosity due to these intergranular voids is denoted by $f_{e}$ (see $\left.(3)\right)$. All voids are assumed to have the same aspect ratio $w(w<1$ for oblate spheroids).

Using the trial field (14), Vincent et al. (2009a) have derived an upper bound for the plastic potential of a single hollow ellipsoid in a Gurson matrix. Then, averaging over all possible orientations of the hollow ellipsoids (as schematically depicted in Figure 3), the effective dissipation potential of an assemblage of hollow ellipsoids has been bounded from above by: 

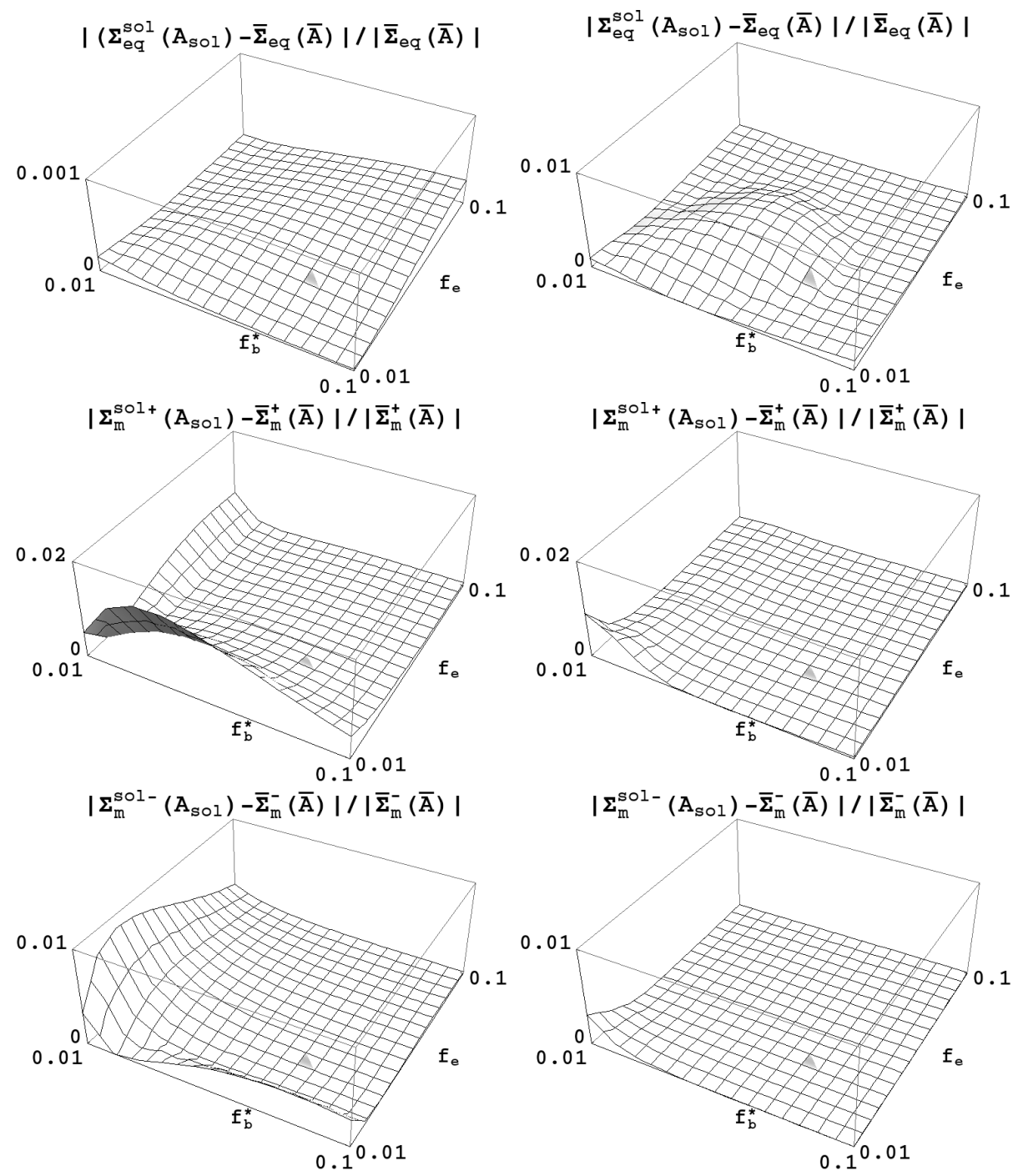

Figure 8: Spherical intergranular voids. $p_{e}=0$ and $q_{3}=1$. Left: $p_{b}=\sigma_{0}$, right: $p_{b}=2 \sigma_{0}$. Comparison between $\Sigma_{m}^{\mathrm{sol} \pm}, \Sigma_{e q}^{\mathrm{sol}}$ obtained with $A_{\text {sol }}$ and $\bar{\Sigma}_{m}^{ \pm}, \bar{\Sigma}_{e q}$ obtained with $\bar{A}$.

$\Phi^{+}\left(\dot{\boldsymbol{E}}, p_{b}, p_{e}\right)=\inf _{A}\left[\sigma_{0} \int_{f_{b}^{*}}^{1} \int_{\lambda_{1}}^{\lambda_{2}} H\left(\lambda, A, y, \dot{E}_{m}, \dot{E}_{e q}\right) d \lambda d y-3\left(1-f_{e}\right) p A\right]-3 p_{e} \dot{E}_{m}$

where

$$
H\left(\lambda, A, y, \dot{E}_{m}, \dot{E}_{e q}\right)=\sqrt{J^{2}(\lambda) \frac{4 A^{2}}{y^{2}}+\frac{J(\lambda)}{q_{3}}\left(3 Q_{J}(\lambda)\left(\dot{E}_{m}-A\right)^{2}+J(\lambda) \dot{E}_{e q}^{2}\right)},
$$

$\lambda$ is related to the systems of ellipsoidal coordinates (see Appendix E). This expression is hardly amenable to analytical calculations. However, following the notations 
and the analysis of Gologanu et al. (1994), Vincent et al. (2008) have noticed that, the potential $\Phi^{+}\left(\dot{\boldsymbol{E}}, p_{b}, p_{e}\right)$ can be approximated with a very good accuracy by a simpler expression

$$
\Phi^{+}\left(\dot{\boldsymbol{E}}, p_{b}, p_{e}\right) \approx \tilde{\Phi}\left(\dot{\boldsymbol{E}}, p_{b}, p_{e}\right)=\inf _{A}\left[\tilde{\phi}\left(\dot{\boldsymbol{E}}, p_{b}, p_{e}, A\right)\right]-3 p_{e} \dot{E}_{m}
$$

where:

$$
\begin{gathered}
\tilde{\phi}\left(\dot{\boldsymbol{E}}, p_{b}, p_{e}, A\right)=\cdots \\
\sigma_{0}(g+1) \int_{f_{b}^{*}}^{1} \int_{\tilde{f}}^{1} \sqrt{\frac{4 A^{2}}{y^{2}}+\frac{\left(\dot{E}_{m}-A\right)^{2}}{z^{2}} \frac{\tilde{a}^{2}}{q_{3}}+\left(\dot{E}_{m}-A\right)^{2} \frac{\tilde{b}^{2}}{q_{3}}+\frac{\left(\dot{E}_{e q}\right)^{2}}{q_{3}}} d z d y \cdots \\
-3\left(1-f_{e}\right) p A .
\end{gathered}
$$

The scalar coefficients $g, \tilde{a}, \tilde{b}$, given by relations (E.8) and (E.11) in Appendix E, depend on the geometry of the ellipsoidal voids only. $\tilde{f}$ is a function of the geometry of the voids and of the actual porosity $f_{e}$ (see (E.8)). The form (43) is very close to the form (17) of the potential for spherical voids where the actual porosity $f_{e}$ of the ellipsoidal voids is replaced by an artificial porosity $\tilde{f}$, except for the presence of the additional term $\left(\dot{E}_{m}-A\right)^{2} \frac{\tilde{b}^{2}}{q_{3}}$. The very same procedure of approximation as for spherical voids can be followed. First an explicit (and accurate) approximation $\bar{A}$ of the average dilatational rate $A_{\text {sol }}$ is proposed, based on specific properties of $A_{\text {sol }}$. Second, this approximation is used to identify the coefficients of a GTN criterion.

\subsection{General properties of $A_{\text {sol }}$}

Let $A_{\mathrm{sol}}(p, \dot{\boldsymbol{E}})$ denote the solution of the variational problem (42). Then the five properties of $A_{\text {sol }}$ that were found for spherical voids have counterparts for ellipsoidal voids, with only slight modifications. Properties 1 and 2 are unchanged: $A_{\text {sol }}$ is an odd function of $(p, \dot{\boldsymbol{E}})$ and a nondecreasing function of $p$

$$
A_{\mathrm{sol}}(-p,-\dot{\boldsymbol{E}})=-A_{\mathrm{sol}}(p, \dot{\boldsymbol{E}}), \quad \frac{\partial A_{\mathrm{sol}}}{\partial p}(p, \dot{\boldsymbol{E}}) \geq 0 .
$$

Properties 3, 4 and 5 have to be slightly amended and read for ellipsoidal voids (see Appendix B for a proof):

Property 3':

$$
\left.\begin{array}{l}
A_{\mathrm{sol}}(p, \dot{\boldsymbol{E}}) \sim \pm \sqrt{\frac{\sigma_{0}(g+1)}{12 q_{3}\left(1-f_{e}\right)}} \sqrt{4 \dot{E}_{m}^{2} \widetilde{I}_{1}+\dot{E}_{e q}^{2} \widetilde{I}_{2}} \frac{1}{\sqrt{\tilde{p}^{\infty} \mp p}} \quad \text { when } \quad p \rightarrow \pm \tilde{p}^{\infty}, \\
\tilde{p}^{\infty}=\frac{2}{3} \frac{\sigma_{0}(g+1)}{1-f_{e}} \tilde{I}_{0},
\end{array}\right\}
$$

where $\tilde{I}_{0}, \tilde{I}_{1}$ and $\tilde{I}_{2}$ are scalar functions of $f_{b}^{*}, f_{e}, w$ given by (B.4) and (B.7). Explicit expressions of $\tilde{I}_{0}, \tilde{I}_{1}$, and $\tilde{I}_{2}$ are given in Appendix C. 
Property 4': $A_{\text {sol }}(p, \dot{\boldsymbol{E}})=0$ when $p=\tilde{p}^{0}(\dot{\boldsymbol{E}})$ with

$$
\left.\begin{array}{l}
\tilde{p}^{0}(\dot{\boldsymbol{E}})=\frac{-4 \sigma_{0}(g+1)\left(1-f_{b}^{*}\right)}{3 \sqrt{q_{3}}\left(1-f_{e}\right)} \dot{E}_{m} \int_{\tilde{f}}^{1} \frac{\tilde{c}^{2}(z)}{z^{2}}\left(4 \frac{\dot{E}_{m}^{2} \tilde{c}^{2}(z)}{z^{2}}+\dot{E}_{e q}^{2}\right)^{-1 / 2} d z, \\
\frac{\partial A_{\mathrm{sol}}}{\partial p}\left(\tilde{p}^{0}, \dot{\boldsymbol{E}}\right)=\frac{3\left(1-f_{e}\right)}{4 \sigma_{0}(g+1) \sqrt{q_{3}}\left(1-f_{b}^{*}\right)} \frac{1}{\left[\frac{1}{f_{b}^{*}}\left(4 \dot{E}_{m}^{2} \tilde{J}_{1}+\dot{E}_{e q}^{2} \tilde{J}_{2}\right)+\frac{\dot{E}_{e q}^{2}}{q_{3}} \tilde{J}_{1}\right]},
\end{array}\right\}
$$

where $\tilde{J}_{1}$ and $\tilde{J}_{2}$ are functions of $f_{b}^{*}, f_{e}, w$ and $\dot{\boldsymbol{E}}$ defined in (B.9).

Property 5': $A_{\text {sol }}(p, \dot{\boldsymbol{E}})=\dot{E}_{m}$ when $p=\tilde{p}^{1}(\dot{\boldsymbol{E}})$ with

$$
\left.\begin{array}{l}
\tilde{p}^{1}(\dot{\boldsymbol{E}})=\frac{4 \sigma_{0}(g+1)}{3} \frac{1-\tilde{f}}{1-f_{e}} \dot{E}_{m} \int_{f_{b}^{*}}^{1} \frac{1}{y^{2}}\left(4 \frac{\dot{E}_{m}^{2}}{y^{2}}+\frac{\dot{E}_{e q}^{2}}{q_{3}}\right)^{-1 / 2} d y, \\
\frac{\partial A_{s o l}}{\partial p}\left(\tilde{p}^{1}, \dot{\boldsymbol{E}}\right)=\frac{3 q_{3}}{4 \sigma_{0}(g+1)} \frac{1-f_{e}}{1-\tilde{f}} \frac{1}{\left[\frac{1}{4}\left(\frac{\tilde{a}^{2}}{\tilde{f}}+\tilde{b}^{2}\right) J_{3}+\dot{E}_{e q}^{2} J_{4}\right]} .
\end{array}\right\}
$$

where $J_{3}$ and $J_{4}$ given by (A.17) are the same as for spherical voids.

\subsection{Explicit approximation for $A_{\text {sol }}$}

An interpolation, matching the above exact asymptotic results, can be proposed in the same vein as in section 3.3. Restricting attention to $\dot{E}_{m}>0$ (the expression of $A_{\text {sol }}$ for $\dot{E}_{m}<0$ being deduced from that for $\dot{E}_{m}>0$ by changing the sign of $p$ and the sign of $A_{\text {sol }}$ ) the interpolation consists of three branches and has the same form as in (27):

$$
\tilde{A}(\dot{\boldsymbol{E}}, p)=\left\{\begin{array}{l}
-\tilde{C}_{-}\left(\frac{\sqrt{\tilde{p}^{\infty}+\tilde{p}^{0}}}{\sqrt{\tilde{p}^{\infty}+p}}-1\right)+\tilde{D}_{-}\left(p-\tilde{p}^{0}\right) \quad \text { when } \quad-\tilde{p}^{\infty}<p \leq \tilde{p}^{0}, \\
\left(p-\tilde{p}^{0}\right)\left(\frac{\tilde{E}}{2}\left(p-\tilde{p}^{1}\right)^{2}+\tilde{F}\left(p-\tilde{p}^{1}\right)+\frac{\dot{E}_{m}}{\tilde{p}^{1}-\tilde{p}^{0}}\right) \quad \text { when } \quad \tilde{p}^{0} \leq p \leq \tilde{p}^{1}, \\
\tilde{C}_{+}\left(\frac{\sqrt{\tilde{p}^{\infty}-\tilde{p}^{1}}}{\sqrt{\tilde{p}^{\infty}-p}}-1\right)+\tilde{D}_{+}\left(p-\tilde{p}^{1}\right)+\dot{E}_{m} \quad \text { when } \quad \tilde{p}^{1} \leq p<\tilde{p}^{\infty},
\end{array}\right.
$$

with

$$
\left.\begin{array}{l}
\tilde{C}_{-}=\frac{1}{\sqrt{\tilde{p}^{\infty}+\tilde{p}^{0}}} \sqrt{\frac{\sigma_{0}(g+1)}{12 q_{3}\left(1-f_{e}\right)}} \sqrt{4 \dot{E}_{m}^{2} \tilde{I}_{1}+E_{e q}^{2} \tilde{I}_{2}}, \\
\tilde{C}_{+}=\frac{1}{\sqrt{\tilde{p}^{\infty}-\tilde{p}^{1}}} \sqrt{\frac{\sigma_{0}(g+1)}{12 q_{3}\left(1-f_{e}\right)}} \sqrt{4 \dot{E}_{m}^{2} \tilde{I}_{1}+E_{e q}^{2} \tilde{I}_{2}},
\end{array}\right\}
$$

The corresponding coefficients $\tilde{D}_{-}, \tilde{D}_{+}, \tilde{E}$ and $\tilde{F}$ are given by (29), (31) and (33) where $p^{0}, p^{1}, p^{\infty}, C_{-}, C_{+}$should be replaced by $\tilde{p}^{0}, \tilde{p}^{1}, \tilde{p}^{\infty}, \tilde{C}_{-}, \tilde{C}_{+}$and where the derivatives $\partial A_{\mathrm{sol}} / \partial p$ at $\tilde{p}^{0}$ and $\tilde{p}^{1}$ are obtained by means of (45) and (46). 


\subsection{Accuracy of the approximation of $\Phi^{+}$by $\tilde{\Phi}$}

In the case of drained voids (no internal pressure) the accuracy of the approximation of $\Phi^{+}$by $\tilde{\Phi}$ has been discussed in Vincent et al. (2008) to which the reader is referred for more details. When the voids are pressurized, we illustrate here the accuracy of this approximation by comparing the limit pressure (beyond which no macroscopic stress can be sustained by the porous material) as predicted by $\Phi^{+}$and by $\tilde{\Phi}$.

It is readily seen that the limit pressure for $\Phi^{+}$is given by:

$$
p_{+}^{\infty}=\frac{1}{3\left(1-f_{e}\right)} \sigma_{0} \int_{f_{b}^{*}}^{1} \int_{\lambda_{1}}^{\lambda_{2}} \sqrt{J^{2}(\lambda) \frac{4}{y^{2}}+\frac{J(\lambda)}{q_{3}} 3 Q_{J}(\lambda)} d \lambda d y .
$$

whereas the limit pressure for $\tilde{\Phi}$ is found to be:

$$
\tilde{p}^{\infty}=\frac{1}{3\left(1-f_{e}\right)} \sigma_{0}(g+1) \int_{f_{b}^{*}}^{1} \int_{\tilde{f}}^{1} \sqrt{\frac{4}{y^{2}}+\frac{1}{z^{2}} \frac{\tilde{a}^{2}}{q_{3}}+\frac{\tilde{b}^{2}}{q_{3}}} d z d y .
$$

$p_{+}^{\infty}$ and $\tilde{p}^{\infty}$ are compared in Figure (9). As can be seen the agreement is very good over a large range of $\left(f_{b}^{*}, f_{e}\right)$ and even for small $w$ and the error is less than $1.5 \%$.

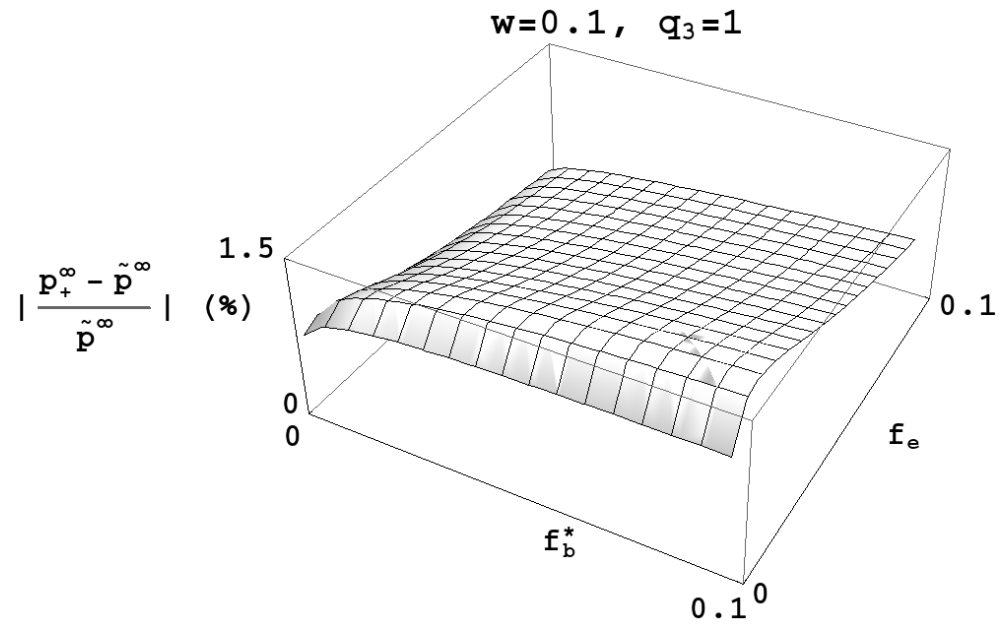

Figure 9: Accuracy of the approximation of the limit pressure between $p_{+}^{\infty}(49)$ by $\tilde{p}^{\infty}(50)$.

\subsection{Comparison between the exact minimizer $A_{\text {sol }}$ and the approximation $\tilde{A}$ (47)}

The exact minimizer $A_{\text {sol }}$ and the approximation $\tilde{A}(47)$ are compared over the whole range of admissible pressures for three specific values of the triaxiality ratio of the overall strain-rate: $\dot{E}_{m} / \dot{E}_{e q}=0, \dot{E}_{m} / \dot{E}_{e q}=+\infty$ and $\dot{E}_{m} / \dot{E}_{e q}=1$. 
5.5.1. Purely deviatoric macroscopic strain-rate: $\dot{E}_{m}=0$.

When the overall strain-rate is purely deviatoric, the approximation (47) takes a simple form with the help of the detailed expressions in Appendix B.1. Four different values of the volume fraction of both populations of voids are considered in Figure 10. As can be seen, the agreement between the exact minimizer of (40) (crosses) and the approximation (47) (solid line) is excellent over the whole range of admissible pressures $p$, and for the four values of the porosities.

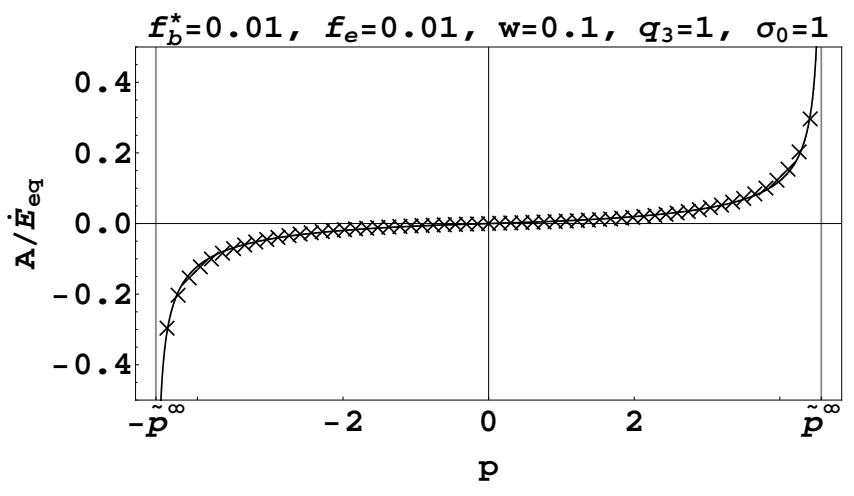

(a)

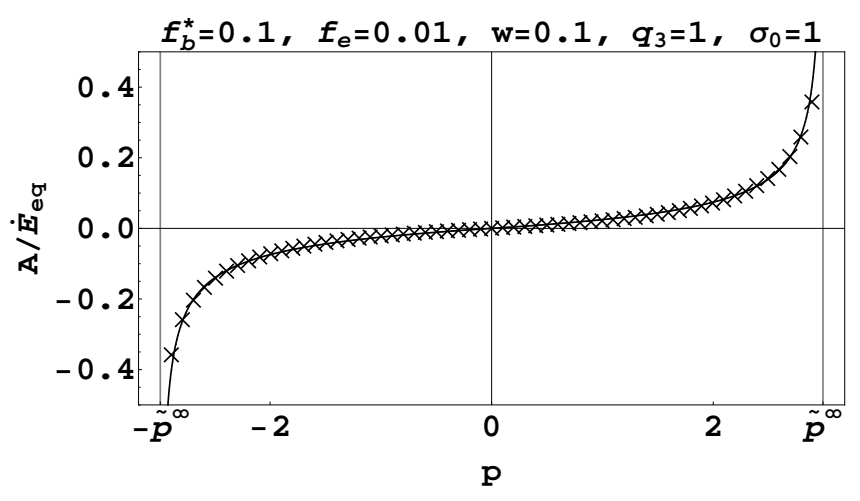

(c)

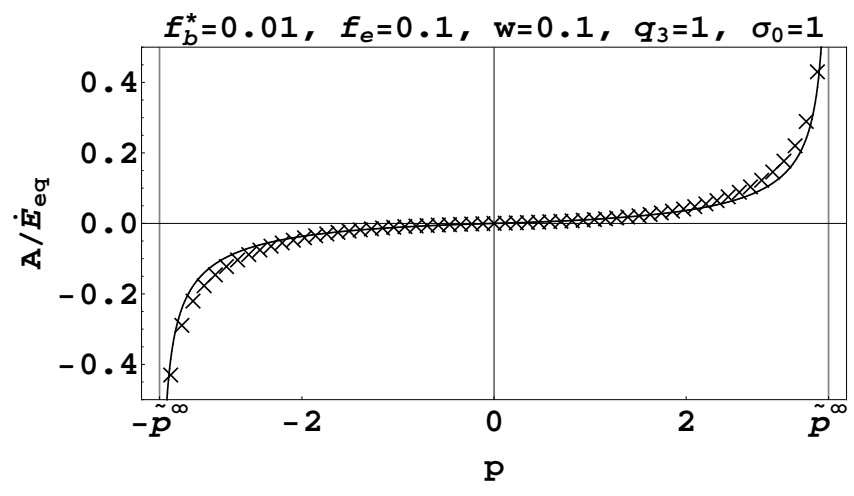

(b)

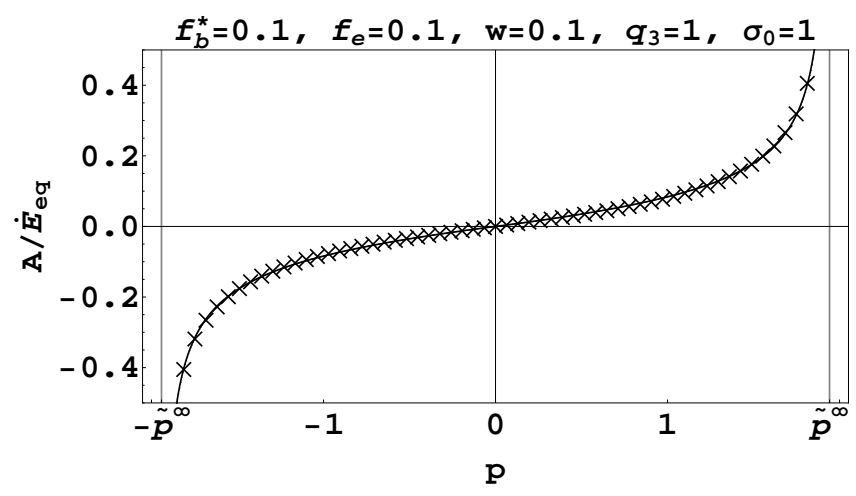

(d)

Figure 10: Ellipsoidal intergranular voids. Purely deviatoric overall strain-rate $\dot{E}_{m}=0$. Comparison between the exact minimizer $A_{\text {sol }}$ for $\Phi^{+}$(crosses) and the approximation $\tilde{A}$ (47) (solid line).

\subsubsection{Purely hydrostatic macroscopic strain-rate: $\dot{E}_{e q}=0$.}

When the overall strain-rate is purely hydrostatic, the two intermediate pressures $\tilde{p}_{0}$ and $\tilde{p}_{1}$ can be computed explicitly (see Appendix B.2). Other simplifications occur in the different terms of the approximation (47) which takes a simplified form thanks to the expressions given in Appendix B.2. Again, the agreement between the exact minimizer of (40) (crosses) and the approximation (47) (solid line) is 
excellent over the whole range of admissible pressures $p$, and for the four values of the porosities (Figure 11).

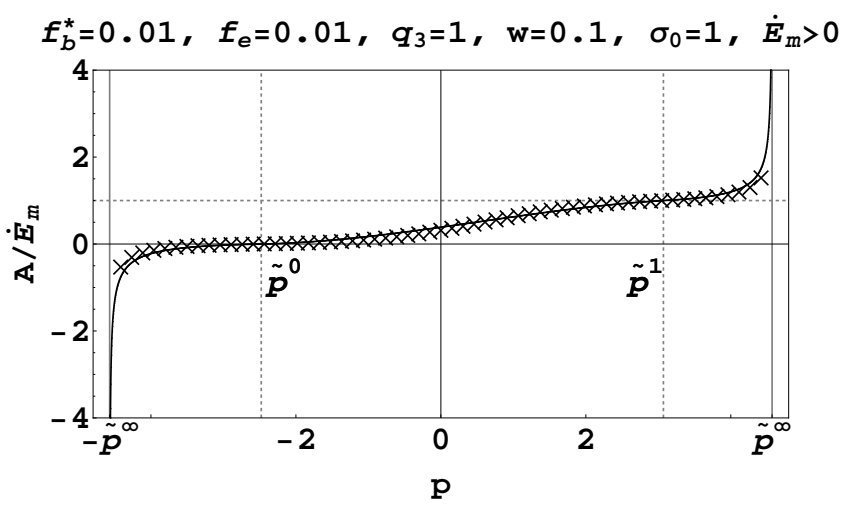

(a)

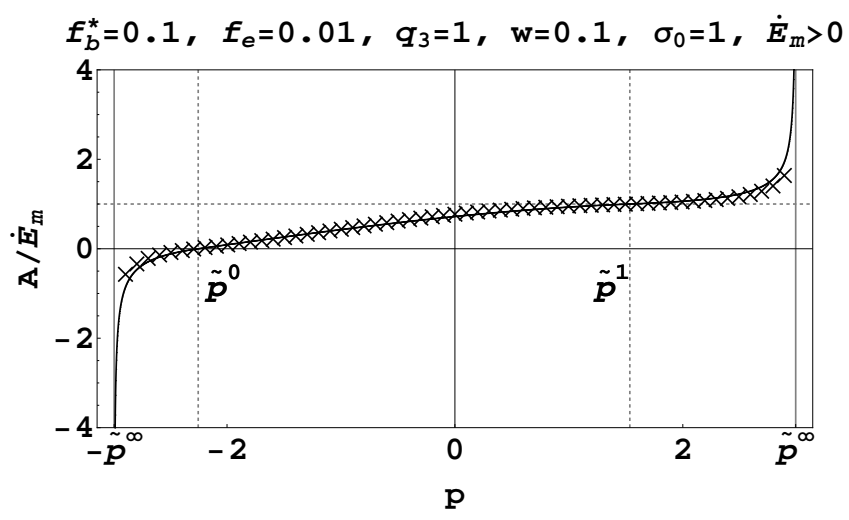

(c)

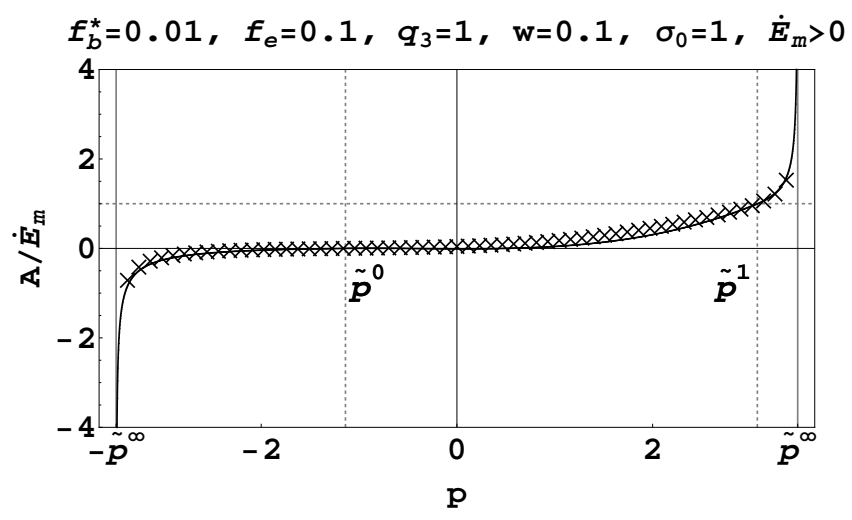

(b)

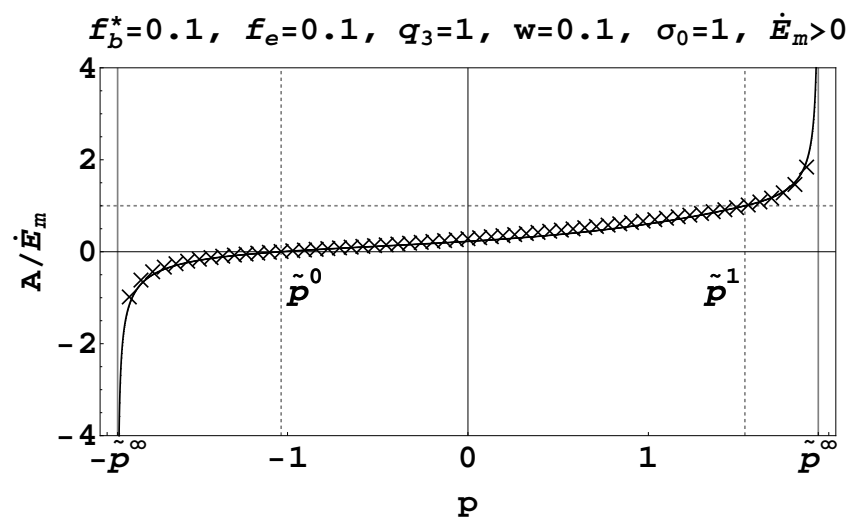

(d)

Figure 11: Ellipsoidal intergranular voids. Purely hydrostatic overall strain-rate $\dot{E}_{e q}=0, \dot{E}_{m}>0$. Comparison between the exact minimizer $A_{\text {sol }}$ for $\Phi^{+}$(crosses) and the approximation $\tilde{A}$ (47) (solid line).

\subsubsection{Intermediate strain-rate: $\dot{E}_{m} / \dot{E}_{e q}=1$.}

For all intermediate values of the triaxiality ratio of the overall strain-rate, the integrals have to be computed numerically. A specific triaxiality ratio $\dot{E}_{m} / \dot{E}_{e q}=1$ has been considered for the comparison performed in Figure 12. Again the agreement between the exact minimizer and its approximation is seen to be excellent.

\subsection{A GTN criterion for pressurized voided materials with ellipsoidal intergranular voids}

It remains to find, as in the spherical case, an accurate approximation for the effective flow surface. First of all, the exact minimizer of (40) leads to an upper 


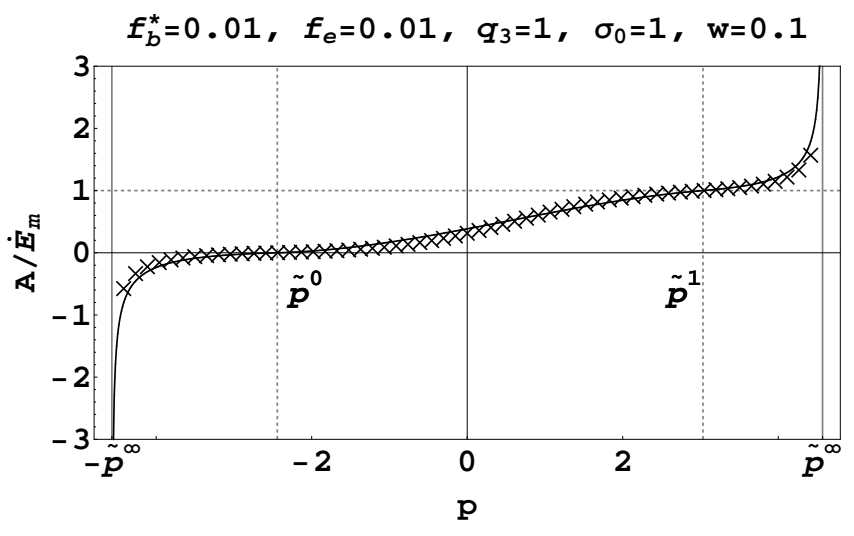

(a)

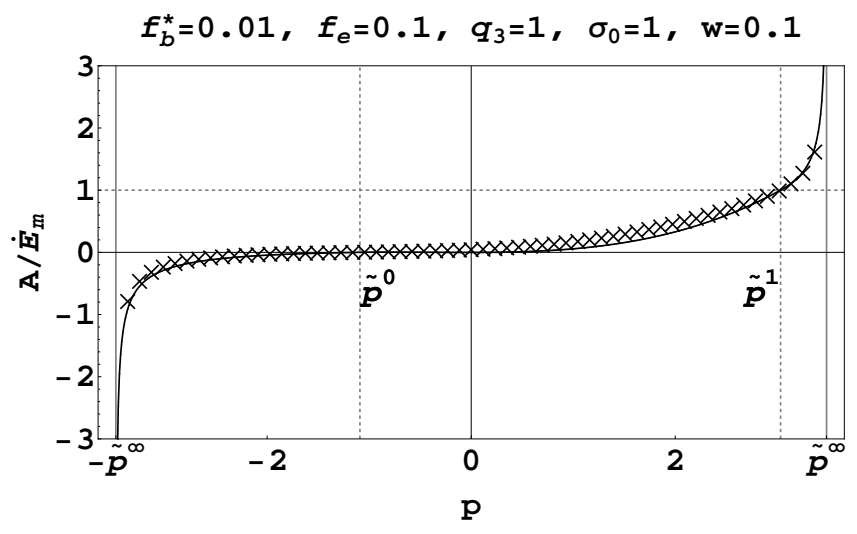

(c)

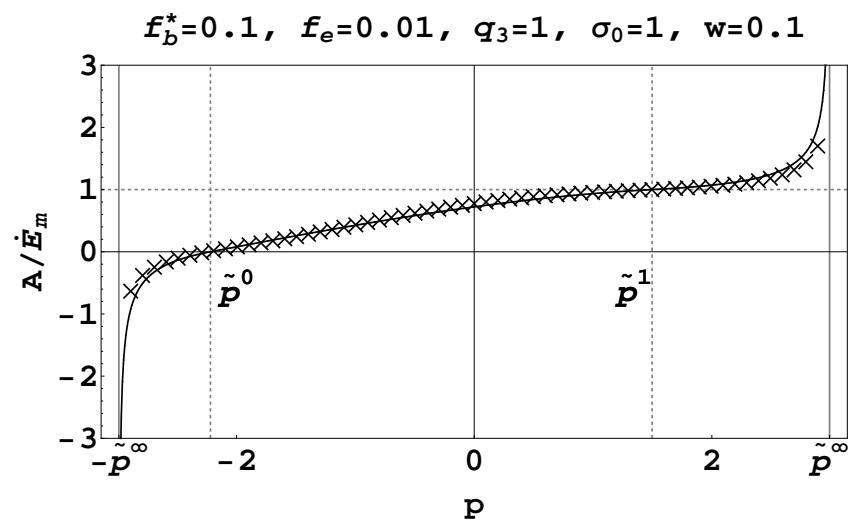

(b)

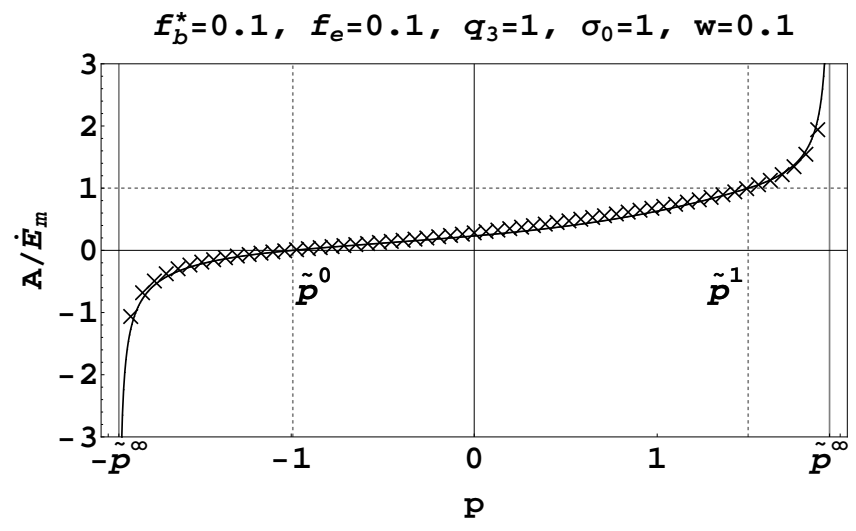

(d)

Figure 12: Ellipsoidal intergranular voids. Triaxial overall strain-rate $\dot{E}_{m} / \dot{E}_{e q}=1$. Comparison between the exact minimizer $A_{\text {sol }}$ for $\Phi^{+}$(crosses) and the approximation $\tilde{A}$ (47) (solid line). 
bound defined by the parametric equations:

$$
\left.\begin{array}{c}
\Sigma_{m}=\frac{1}{3} \frac{\partial \Phi^{+}}{\partial \dot{E}_{m}}\left(\dot{\boldsymbol{E}}, p_{b}, p_{e}\right), \quad \Sigma_{e q}=\frac{\partial \Phi^{+}}{\partial \dot{E}_{e q}}\left(\dot{\boldsymbol{E}}, p_{b}, p_{e}\right), \\
\Sigma_{m}=-p_{e}+\frac{\sigma_{0}}{q_{3}} \int_{f_{b}^{*}}^{1} \int_{\lambda_{1}}^{\lambda_{2}} \frac{J(\lambda) Q_{J}(\lambda)}{H\left(\lambda, A_{\mathrm{sol}}, y, \dot{E}_{m}, \dot{E}_{e q}\right)} d \lambda d y\left(\dot{E}_{m}-A_{\mathrm{sol}}\right), \\
\Sigma_{e q}=\frac{\sigma_{0}}{q_{3}} \int_{f_{b}^{*}}^{1} \int_{\lambda_{1}}^{\lambda_{2}} \frac{J^{2}(\lambda)}{H\left(\lambda, A_{\mathrm{sol}}, y, \dot{E}_{m}, \dot{E}_{e q}\right)} d \lambda d y \dot{E}_{e q} .
\end{array}\right\}
$$

To simplify these relations a first approach consists in using the approximation $\tilde{A}$ in (47) to get an upper bound, denoted by $\bar{\Phi}$ for the overall dissipation potential $\Phi^{+}$:

$$
\begin{aligned}
\bar{\Phi}\left(\dot{\boldsymbol{E}}, p_{b}, p_{e}\right)= & \sigma_{0} \int_{f_{b}^{*}}^{1} \int_{\lambda_{1}}^{\lambda_{2}} H\left(\lambda, \tilde{A}\left(\dot{E}_{m}, \dot{E}_{e q}\right), y, \dot{E}_{m}, \dot{E}_{e q}\right) d \lambda d y \\
& -3\left(1-f_{e}\right) p \tilde{A}\left(\dot{E}_{m}, \dot{E}_{e q}\right)-3 p_{e} \dot{E}_{m} .
\end{aligned}
$$

The associated flow surface is then an upper bound defined by the parametric equations (35). It is recalled here that the terms proportional to the derivatives of $\tilde{A}$ with respect to $\dot{E}_{m}$ and $\dot{E}_{e q}$ have to be properly taken into account in the expressions $(35)$.

The two flow surfaces, which remain two upper bounds, are compared in Figure 13. The agreement between crosses $\left(\Phi^{+}\right)$and squares $(\bar{\Phi})$ is seen to be excellent. This proves that the expression (47) is an accurate approximation for the exact minimizer of (40). However, even if the approximation of $\Phi^{+}$by $\bar{\Phi}$ avoids the resolution of an optimization problem to determine $A_{\text {sol }}$, the evaluation of the double integrals in the analog of (51) (with $\bar{\Phi}$ instead of $\Phi^{+}$) is still time-consuming when repeated at each Gauss point of a structure. This motivates the search of an explicit equation for the effective flow surface.

Following section 4, a GTN flow surface in the form (1) is proposed, based on $\tilde{A}$ (47) and $\tilde{\phi}(43)$. The three specific stresses $\bar{\Sigma}_{m}^{-}, \bar{\Sigma}_{m}^{+}, \bar{\Sigma}_{e q}$ which specify completely the form (1) and correspond respectively to the minimum and maximum hydrostatic stresses and to the maximum deviatoric stress on the flow surface are then given by:

$\bar{\Sigma}_{m}^{ \pm}= \pm \frac{1}{3} \tilde{\phi}\left(\tilde{A}^{ \pm}, \pm \boldsymbol{i}, p\right)-p_{e}, \quad \tilde{A}^{ \pm}=\tilde{A}( \pm \boldsymbol{i}, p), \quad \bar{\Sigma}_{e q}=\tilde{\phi}\left(\tilde{A}_{e q}, \dot{\boldsymbol{E}}^{d}, p\right), \quad \tilde{A}_{e q}=\tilde{A}\left(\dot{\boldsymbol{E}}^{d}, p\right)$,

where $\tilde{A}^{ \pm}$and $\tilde{A}_{e q}$ can be expressed in closed form by means of (Appendix B.1) and (Appendix B.2). The relations (54), together with the definition (43) of $\tilde{\phi}$ lead 
to:

$$
\begin{aligned}
\bar{\Sigma}_{m}^{ \pm}= & \pm \frac{1}{3} \sigma_{0}(g+1) \int_{f_{b}^{*}}^{1} \int_{\tilde{f}}^{1} \sqrt{\frac{4\left(\tilde{A}^{ \pm}\right)^{2}}{y^{2}}+\frac{\left( \pm 1-\tilde{A}^{ \pm}\right)^{2}}{z^{2}} \frac{\tilde{a}^{2}}{q_{3}}+\left( \pm 1-\tilde{A}^{ \pm}\right)^{2} \frac{\tilde{b}^{2}}{q_{3}}} d z d y \\
& -\left(1-f_{e}\right) p \tilde{A}^{ \pm}-p_{e}, \\
\bar{\Sigma}_{e q}= & \sigma_{0}(g+1) \int_{f_{b}^{*}}^{1} \int_{\tilde{f}}^{1} \sqrt{\frac{4\left(\tilde{A}_{e q}\right)^{2}}{y^{2}}+\frac{\left(\tilde{A}_{e q}\right)^{2}}{z^{2}} \frac{\tilde{a}^{2}}{q_{3}}+\left(\tilde{A}_{e q}\right)^{2} \frac{\tilde{b}^{2}}{q_{3}}+\frac{1}{q_{3}}} d z d y \\
& -3\left(1-f_{e}\right) p \tilde{A}_{e q} .
\end{aligned}
$$

The integrals entering (55) can be expressed in closed form (see Appendix C). The three flow surfaces corresponding to the two upper bounds (35) and (52), and the approximation (1) are compared in Figure 13. As can be seen the agreement is very satisfactory at all stress triaxiality.

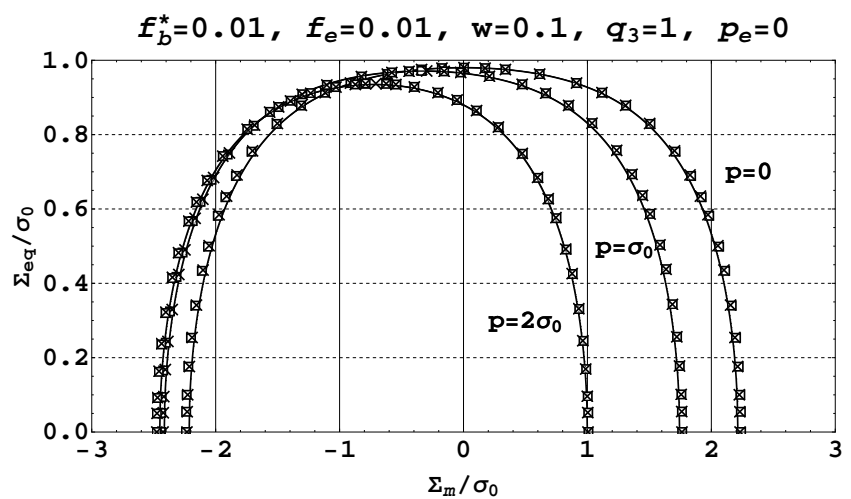

(a)

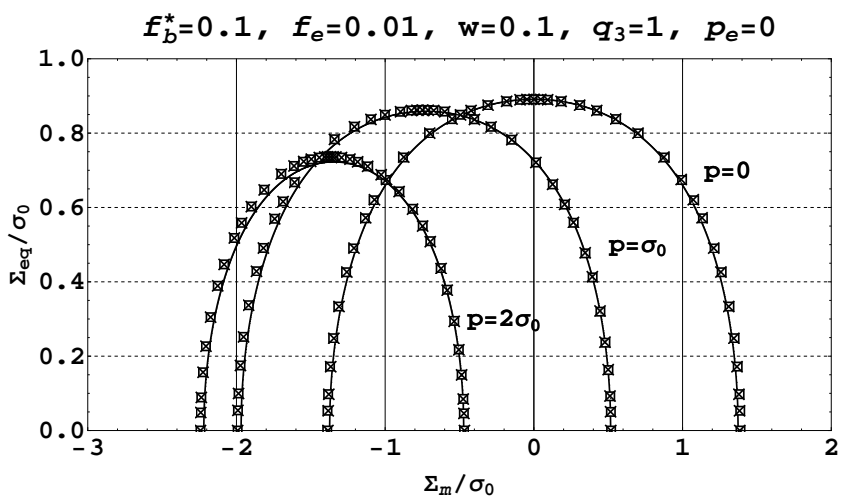

(c)

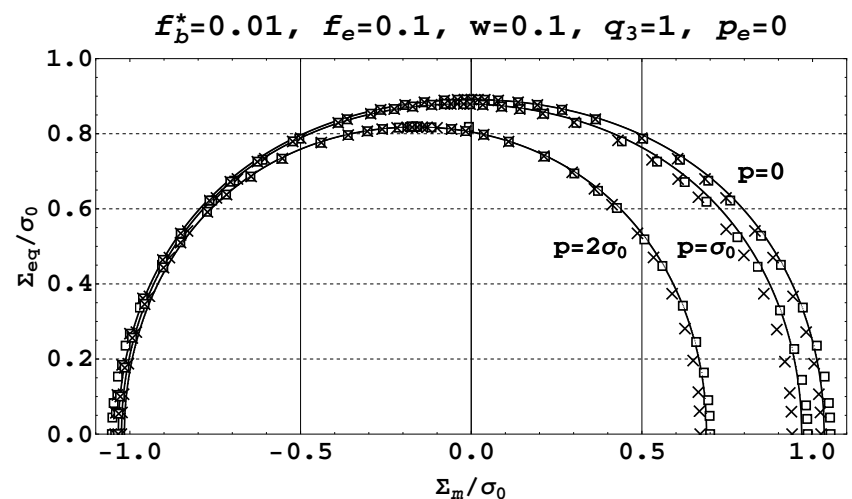

(b)

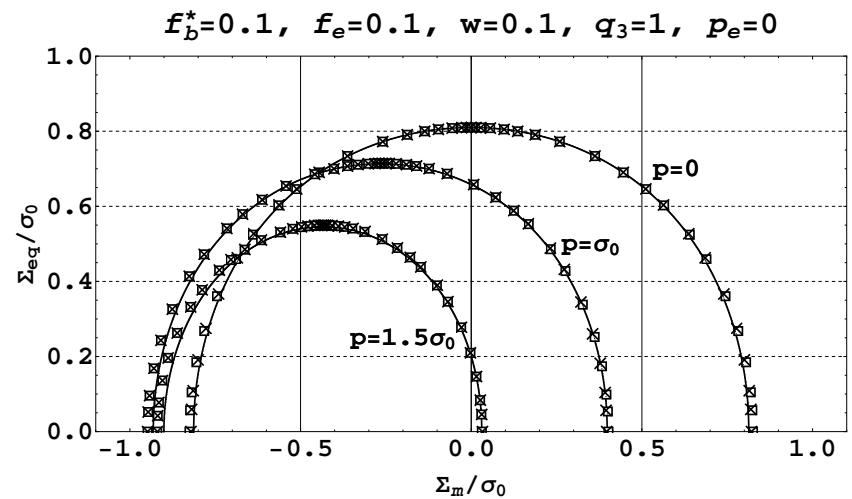

(d)

Figure 13: Ellipsoidal intergranular voids. Comparison between the flow surface (52) associated with $\Phi^{+}(40)$ (crosses), the flow surface (35) associated with $\bar{\Phi}$ (53) (squares) and the GTN approximation (1) (solid line). $p$ denotes the difference $p_{b}-p_{e}$. 


\section{Conclusion}

This study is devoted to porous materials with two populations of randomly distributed voids with different sizes. The smallest voids are spherical whereas the largest voids are spheroidal. Both population of voids are subjected to an internal homogeneous pressure. In this study, attention has been focused on analytical approximations for the dilatation-rate in the matrix at the mesoscopic scale and for the effective flow surface at the macroscopic scale. An analytical estimate for the effective flow surface, obtained by extending the result of Vincent et al. (2008) to saturated voids has been proposed. This new criterion has the same form as the classical GTN criterion, which facilitates its integration in any standard FEM code in which the GTN criterion is already implemented. In the second part of this study (next paper) the accuracy of the new model is assessed by comparison with full field numerical simulations.

Acknowledments: H. Moulinec and P. Suquet acknowledge the financial support of Labex MEC and of A*Midex through grants ANR-11-LABX-0092 and ANR-11IDEX-0001-02. 


\section{References}

Benzerga, A., Besson, J., Pineau, A., 2004. Anisotropic ductile fracture, part II: theory. Acta Materialia 152, 4639-4650.

Bilger, N., Auslender, F., Bornert, M., Masson, R., 2002. New bounds and estimates for porous media with rigid perfectly plastic matrix. Comptes Rendus Mécanique $330,127-132$.

Danas, K., Idiart, M., Ponte Castañeda, P., 2008. A homogenization-based constitutive model for isotropic viscoplastic porous media. International Journal of Solids and Structures 45, 3392-3409.

Dherbey, F., Louchet, F., A. Mocellin, A., Leclercq, S., 2002. Elevated temperature creep of polycrystalline uranium dioxide : from microscopic mechanisms to macroscopic behaviour. Acta Materialia 50, 1495-1505.

Dubourg, R., Faure-Geors, H., Nicaise, G., Barrachin, M., 2005. Fission product release in the first two PHEBUS tests FPT0 and FPT1. Nuclear Engineering and Design 235, 2183-2208.

Fabrègue, D., Pardoen, T., 2008. A constitutive model for elastoplastic solids containing primary and secondary voids. Journal of the Mechanics and Physics of Solids 56, 719-741.

Gologanu, M., Leblond, J., Devaux, J., 1994. Approximate models for ductile metals containing non-spherical voids-case of axisymmetric oblate ellipsoidal cavities. ASME Journal of Engineering Materials and Technology 116, 290-297.

Gologanu, M., Leblond, J., Perrin, G., Devaux, J., 1997. Recent extensions of Gurson's model for porous ductile metals. In: Suquet, P. (Ed.), Continuum Micromechanics. Springer-Verlag, New-York, pp. 61-130.

Guo, T., Faleskog, J., Shih, C., 2008. Continuum modeling of a porous solid with pressure sensitive dilatant matrix. Journal of the Mechanics and Physics of Solids 56, 2188-2212.

Gurson, A., 1977. Continuum theory of ductile rupture by void nucleation and growth: Part I - Yield criteria and flow rules for porous ductile media. Journal of Engineering Materials and Technology 99, 1-15.

Julien, J., Gărăjeu, M., Michel, J.-C., 2011. A semi-analytical model for the behavior of saturated viscoplastic materials containing two populations of voids of different sizes. International Journal of Solids and Structures 48, 1485-1498.

Kashibe, S., Une, K., 1991. Effects of temperature cycling and heating rate on fission gas release of BWR fuels. Journal of Nuclear Science and Technology 28, 1090-1099. 
Kashibe, S., Une, K., Nogita, K., 1993. Formation and growth of intragranular fission gas bubbles in $\mathrm{UO}_{2}$ fuels with burnup of $6-83 \mathrm{GWd} / \mathrm{t}$. Journal of Nuclear Materials 206, 22-34.

Koplik, J., Needleman, A., 1988. Void growth and coalescence in porous plastic solids. International Journal of Solids and Structures 24, 835-853.

Lebensohn, R.A., Idiart, M.I., Ponte Castañeda, P., Vincent, P.G., 2011. Dilatational viscoplasticity of polycrystalline solids with intergranular cavities. Philosophical Magazine 91, 3038-3067.

Leblond, J., Perrin, G., 1999. A self-consistent approach to coalescence of cavities in inhomogeneously voided ductile solids. Journal of the Mechanics and Physics of Solids 47, 1823-1841.

Leblond, J., Perrin, G., Suquet, P., 1994. Exact results and approximate models for porous viscoplastic solids. International Journal of Plasticity 10, 213-235.

Lee, J., Oung, J., 1999. Yield functions and flow rules for porous pressure-dependent strain-hardening polymeric materials. Journal of Applied Mechanics 67, 288-297.

Lösönen, P., 2000. On the behaviour of intragranular fission gas in $\mathrm{UO}_{2}$ fuel. Journal of Nuclear Materials 280, 56-72.

Madou, K., Leblond, J., 2012. A Gurson-type criterion for porous ductile solids containing arbitrary ellipsoidal voids I: Limit-analysis of some representative cell. Journal of the Mechanics and Physics of Solids 60, 1020-1036.

Michel, J., Moulinec, H., Suquet, P., 2000. A computational method based on augmented Lagrangians and fast Fourier transforms for composites with high contrast. Computer Modeling in Engineering and Sciences 1 (2), 79-88.

Michel, J., Moulinec, H., Suquet, P., 2001. A computational method for linear and nonlinear composites with arbitrary phase contrast. International Journal for $\mathrm{Nu}-$ merical Methods in Engineering 52, 139-160.

Monchiet, V., Charkaluk, E., Kondo, D., 2011. A micromechanics-based modification of the Gurson criterion by using Eshelby-like velocity fields. European Journal of Mechanics A/Solids 30, 940-949.

Monchiet, V., Kondo, D., 2012. Exact solution of a plastic hollow sphere with a Mises-Schleicher matrix. International Journal of Engineering Science 51, 168 178.

Moulinec, H., Suquet, P., 1998. A numerical method for computing the overall response of nonlinear composites with complex microstructure. Computer Methods in Applied Mechanics and Engineering 157, 69-94. 
Olander, D., 1976. Fundamental Aspects of Nuclear Reactor Fuel Elements. Technical Information Center, Office of Public Affairs. Energy Reasearch and Development Administration. TID-26711-P1.

Ponte Castañeda, P., 1991. The effective mechanical properties of nonlinear isotropic composites. Journal of the Mechanics and Physics of Solids 39, 45-71.

Ponte Castañeda, P., Suquet, P., 1998. Nonlinear composites. Advances in Applied Mechanics 34, 171-302.

Ponte Castañeda, P., Zaidman, M., 1996. The finite deformation of nonlinear composite materials. I. Instantaneous constitutive relations. International Journal of Solids and Structures 33, 1271-1286.

Suquet, P., 1995. Overall properties of nonlinear composites : a modified secant moduli theory and its link with Ponte Castañeda's nonlinear variational procedure. Comptes Rendus Académie des Sciences Paris 320, Série IIb, 563-571.

Thoré, P., Pastor, F., Pastor, J., Kondo, D., 2009. Closed-form solutions for the hollow sphere model with Coulomb and Drucker-Prager materials under isotropic loadings. Comptes Rendus Mécanique 337 (5), 260-267.

Tvergaard, V., 1990. Material failure by void growth to coalescence. In: Hutchinson, J., Wu, T. (Eds.), Advances in Applied Mechanics. Vol. 27. Academic Press, NewYork, pp. 83-151.

Vincent, P.-G., Monerie, Y., Suquet, P., 2008. Ductile damage of porous materials with two populations of voids. Comptes Rendus Mécanique 336, 245-259.

Vincent, P.-G., Monerie, Y., Suquet, P., 2009a. Porous materials with two populations of voids under internal pressure: I. Instantaneous constitutive relations. International Journal of Solids and Structures 46, 480-506.

Vincent, P.-G., Monerie, Y., Suquet, P., 2009b. Porous materials with two populations of voids under internal pressure: II. Growth and coalescence of voids. International Journal of Solids and Structures 46, 507-526.

Zaidman, M., Ponte Castañeda, P., 1996. The finite deformation of nonlinear composite materials. II. Evolution of the microstructure. International Journal of Solids and Structures 33, 1287-1303. 


\section{Appendix A. Properties of $\boldsymbol{A}_{\mathrm{sol}}(\dot{E}, p)$ for spherical cavities}

Proof of Property 1: From the definition (17) of $\phi$ it is readily seen that:

$$
\phi(-A,-\dot{\boldsymbol{E}},-p)=\phi(A, \dot{\boldsymbol{E}}, p),
$$

and that the function which is to be minimized in the right-hand side of (16) is also an even function of $\left(A, \dot{\boldsymbol{E}}, p_{b}, p_{e}\right)$. Since it is a strictly convex function of $A$ (whenever $\dot{\boldsymbol{E}} \neq 0$ which is the case throughout the paper), the minimizer $A_{\text {sol }}$ in (16) is unique. Now consider the minimization problem for $\left(-\dot{\boldsymbol{E}},-p_{b},-p_{e}\right)$. Its unique minimizer $A_{\mathrm{sol}}(-\dot{\boldsymbol{E}},-p)$ satisfies

$$
\phi\left(A_{\mathrm{sol}}(-\dot{\boldsymbol{E}},-p),-\dot{\boldsymbol{E}},-p\right) \leq \phi(B,-\dot{\boldsymbol{E}},-p) \text { for all } B .
$$

Choosing $B=-A$ and the parity property (A.1) in the two sides of (A.2) shows that

$$
\phi\left(-A_{\mathrm{sol}}(-\dot{\boldsymbol{E}},-p), \dot{\boldsymbol{E}}, p\right) \leq \phi(A, \dot{\boldsymbol{E}}, p) \text { for all } A,
$$

which proves, by virtue of the uniqueness of the minimizer, that

$$
-A_{\text {sol }}(-\dot{\boldsymbol{E}},-p)=A_{\mathrm{sol}}(\dot{\boldsymbol{E}}, p) .
$$

Proof of Property 2: Note that $\phi$ is a convex function of all its arguments $p, A, \dot{\boldsymbol{E}}$ separately. The stationarity of $\phi$ with respect to $A$ implies

$$
\frac{\partial \phi}{\partial A}\left(A_{\mathrm{sol}}, \dot{\boldsymbol{E}}, p\right)=0,
$$

and by derivation with respect to $p$, one gets

$$
\frac{\partial^{2} \phi}{\partial A^{2}}\left(A_{\mathrm{sol}}, \dot{\boldsymbol{E}}, p\right) \frac{\partial A_{\mathrm{sol}}}{\partial p}+\frac{\partial^{2} \phi}{\partial A \partial p}\left(A_{\mathrm{sol}}, \dot{\boldsymbol{E}}, p\right)=0 .
$$

$\phi$ is a convex function of $A$ and $\partial^{2} \phi / \partial A^{2}$ is positive. In addition, it follows from (17) that $-\partial^{2} \phi / \partial A \partial p=3\left(1-f_{e}\right)>0$. Therefore $A_{\text {sol }}$ is a monotone increasing function of $p$.

Proof of Property 3: The function $A_{\text {sol }}(\dot{\boldsymbol{E}}, p)$ can be inverted to get a function $p\left(A_{\text {sol }}, \dot{\boldsymbol{E}}\right)$ which is obtained by writing the optimality condition of $\phi$ with respect to $A$

$$
\frac{\partial \phi}{\partial A}\left(A_{\mathrm{sol}}, \dot{\boldsymbol{E}}, p\right)=0 .
$$

A straightforward calculation gives:

$$
\frac{\partial \phi}{\partial A}(A, \dot{\boldsymbol{E}}, p)=\sigma_{0} \int_{f_{e}}^{1} \int_{f_{b}^{*}}^{1} \frac{4 \frac{A}{y^{2}}+4 \frac{\left(A-\dot{E}_{m}\right)}{q_{3} z^{2}}}{\sqrt{4 \frac{A^{2}}{y^{2}}+4 \frac{\left(\dot{E}_{m}-A\right)^{2}}{q_{3} z^{2}}+\frac{\left(\dot{E}_{e q}\right)^{2}}{q_{3}}}} d y d z-3\left(1-f_{e}\right) p,
$$


and therefore the function $p\left(A_{\text {sol }}, \dot{\boldsymbol{E}}\right)$ reads as:

$$
p\left(A_{\mathrm{sol}}, \dot{\boldsymbol{E}}\right)=\frac{\sigma_{0}}{3\left(1-f_{e}\right)} \int_{f_{e}}^{1} \int_{f_{b}^{*}}^{1} \frac{4 \frac{A_{\mathrm{sol}}}{y^{2}}+4 \frac{\left(A_{\mathrm{sol}}-\dot{E}_{m}\right)}{q_{3} z^{2}}}{\sqrt{4 \frac{A_{\mathrm{sol}}^{2}}{y^{2}}+4 \frac{\left(\dot{E}_{m}-A_{\mathrm{sol}}\right)^{2}}{q_{3} z^{2}}+\frac{\left(\dot{E}_{e q}\right)^{2}}{q_{3}}}} d y d z .
$$

Taking the limit of (A.5) as $A_{\text {sol }} \rightarrow \pm \infty$ yields

$$
\lim _{A \rightarrow \pm \infty} p\left(A_{\mathrm{sol}}, \dot{\boldsymbol{E}}\right)= \pm p^{\infty}, \quad p^{\infty}=\frac{2}{3} \frac{\sigma_{0}}{1-f_{e}} I_{0},
$$

where

$$
I_{0}=\int_{f_{e}}^{1} \int_{f_{b}^{*}}^{1} \sqrt{\frac{1}{y^{2}}+\frac{1}{q_{3} z^{2}}} d y d z
$$

To prove the more specific result (22), use is made of (A.3). A straightforward calculation gives:

$$
\frac{\partial^{2} \phi}{\partial A \partial p}(A, \dot{\boldsymbol{E}}, p)=-3\left(1-f_{e}\right)
$$

and

$$
\frac{\partial^{2} \phi}{\partial A^{2}}(A, \dot{\boldsymbol{E}}, p)=\sigma_{0} \int_{f_{e}}^{1} \int_{f_{b}^{*}}^{1} \frac{\frac{16 \dot{E}_{m}^{2}}{q_{3} y^{2} z^{2}}+\frac{4 \dot{E}_{e q}^{2}}{q_{3}}\left(\frac{1}{y^{2}}+\frac{1}{q_{3} z^{2}}\right)}{\left(4 \frac{A^{2}}{y^{2}}+4 \frac{\left(\dot{E}_{m}-A\right)^{2}}{q_{3} z^{2}}+\frac{\left(\dot{E}_{e q}\right)^{2}}{q_{3}}\right)^{3 / 2}} d y d z
$$

It follows from (A.9) that as $p$ approaches $\pm p^{\infty}$, i.e. when $A_{\text {sol }}$ approaches $\pm \infty$

$$
\frac{\partial^{2} \phi}{\partial A^{2}}\left(A_{\mathrm{sol}}, \dot{\boldsymbol{E}}, p\right) \sim\left|A_{\mathrm{sol}}\right|^{-3} \frac{\sigma_{0}}{2 q_{3}}\left(4 \dot{E}_{m}^{2} I_{1}+\dot{E}_{e q}^{2} I_{2}\right),
$$

where

$$
I_{1}=\int_{f_{e}}^{1} \int_{f_{b}^{*}}^{1} \frac{1}{y^{2} z^{2}}\left(\frac{1}{y^{2}}+\frac{1}{q_{3} z^{2}}\right)^{-3 / 2} d y d z, \quad I_{2}=\int_{f_{e}}^{1} \int_{f_{b}^{*}}^{1}\left(\frac{1}{y^{2}}+\frac{1}{q_{3} z^{2}}\right)^{-1 / 2} d y d z
$$

Therefore, according to (A.3):

$$
\left|A_{\mathrm{sol}}\right|^{-3} \frac{\partial A_{\mathrm{sol}}}{\partial p}(\dot{\boldsymbol{E}}, p) \sim \frac{6 q_{3}\left(1-f_{e}\right)}{\sigma_{0}} \frac{1}{\left(4 \dot{E}_{m}^{2} I_{1}+\dot{E}_{e q}^{2} I_{2}\right)},
$$

which gives by integration between $p$ and $p^{\infty}$

$$
A_{\mathrm{sol}}(p, \dot{\boldsymbol{E}}) \sim \sqrt{\frac{\sigma_{0}}{12 q_{3}\left(1-f_{e}\right)}} \sqrt{4 \dot{E}_{m}^{2} I_{1}+\dot{E}_{e q}^{2} I_{2}} \frac{1}{\sqrt{p^{\infty}-p}} \quad \text { when } \quad p \sim p^{\infty} .
$$


A similar integration can be performed between $-p^{\infty}$ and $p$ to get a similar asymptotic result when $p \sim-p^{\infty}$.

Proof of Property 4: The derivative of $A_{\text {sol }}(p, \dot{\boldsymbol{E}})$ at $p=p^{0}(\dot{\boldsymbol{E}})$ (where $A_{\text {sol }}=0$ ) can be obtained from

$$
\frac{\partial^{2} \phi}{\partial A^{2}}\left(0, \dot{\boldsymbol{E}}, p^{0}\right) \frac{\partial A_{\mathrm{sol}}}{\partial p}\left(p^{0}, \dot{\boldsymbol{E}}\right)=-\frac{\partial^{2} \phi}{\partial A \partial p}\left(0, \dot{\boldsymbol{E}}, p^{0}\right)=3\left(1-f_{e}\right) .
$$

It follows from the general expression (A.9) that

$$
\begin{gathered}
\frac{\partial^{2} \phi}{\partial A^{2}}\left(0, \dot{\boldsymbol{E}}, p^{0}\right)=4 \sigma_{0} \sqrt{q_{3}} \int_{f_{e}}^{1} \int_{f_{b}^{*}}^{1} \frac{\frac{4 \dot{E}_{m}^{2}}{y^{2} z^{2}}+\dot{E}_{e q}^{2}\left(\frac{1}{y^{2}}+\frac{1}{q_{3} z^{2}}\right)}{\left(\frac{4 \dot{E}_{m}^{2}}{z^{2}}+\dot{E}_{e q}^{2}\right)^{3 / 2}} d y d z . \\
=4 \sigma_{0} \sqrt{q_{3}}\left(1-f_{b}^{*}\right)\left[\frac{1}{f_{b}^{*}}\left(4 \dot{E}_{m}^{2} J_{1}+\dot{E}_{e q}^{2} J_{2}\right)+\frac{\dot{E}_{e q}^{2}}{q_{3}} J_{1}\right]
\end{gathered}
$$

where

$$
J_{1}=\int_{f_{e}}^{1} \frac{1}{z^{2}}\left(\frac{4 \dot{E}_{m}^{2}}{z^{2}}+\dot{E}_{e q}^{2}\right)^{-3 / 2} d z, \quad J_{2}=\int_{f_{e}}^{1}\left(\frac{4 \dot{E}_{m}^{2}}{z^{2}}+\dot{E}_{e q}^{2}\right)^{-3 / 2} d z
$$

Therefore

$$
\frac{\partial A_{\mathrm{sol}}}{\partial p}\left(p^{0}, \dot{\boldsymbol{E}}\right)=\frac{3\left(1-f_{e}\right)}{4 \sigma_{0} \sqrt{q_{3}}\left(1-f_{b}^{*}\right)} \frac{1}{\left[\frac{1}{f_{b}^{*}}\left(4 \dot{E}_{m}^{2} J_{1}+\dot{E}_{e q}^{2} J_{2}\right)+\frac{\dot{E}_{e q}^{2}}{q_{3}} J_{1}\right]} .
$$

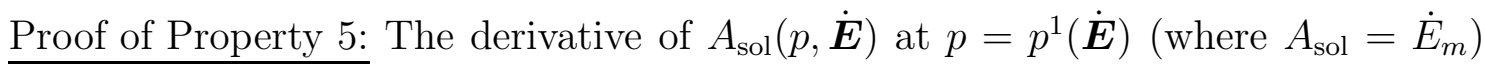
can be obtained from

$$
\frac{\partial^{2} \phi}{\partial A^{2}}\left(\dot{E}_{m}, \dot{\boldsymbol{E}}, p^{1}\right) \frac{\partial A_{\mathrm{sol}}}{\partial p}\left(p^{1}, \dot{\boldsymbol{E}}\right)=-\frac{\partial^{2} \phi}{\partial A \partial p}\left(\dot{E}_{m}, \dot{\boldsymbol{E}}, p^{1}\right)=3\left(1-f_{e}\right) .
$$

It follows from the general expression (A.9) that

$$
\begin{aligned}
\frac{\partial^{2} \phi}{\partial A^{2}}\left(\dot{E}_{m}, \dot{\boldsymbol{E}}, p^{1}\right)= & 4 \frac{\sigma_{0}}{q_{3}} \int_{f_{e}}^{1} \int_{f_{b}^{*}}^{1} \frac{\frac{4 \dot{E}_{m}^{2}}{y^{2} z^{2}}+\dot{E}_{e q}^{2}\left(\frac{1}{y^{2}}+\frac{1}{q_{3} z^{2}}\right)}{\left(\frac{4 \dot{E}_{m}^{2}}{y^{2}}+\frac{\dot{E}_{e q}^{2}}{q_{3}}\right)^{3 / 2}} d y d z . \\
& =4 \frac{\sigma_{0}}{q_{3}}\left(1-f_{e}\right)\left(\frac{1}{f_{e}} J_{3}+\dot{E}_{e q}^{2} J_{4}\right),
\end{aligned}
$$


where

$$
J_{3}=\int_{f_{b}^{*}}^{1}\left(\frac{4 \dot{E}_{m}^{2}}{y^{2}}+\frac{\dot{E}_{e q}^{2}}{q_{3}}\right)^{-1 / 2} d y, \quad J_{4}=\int_{f_{b}^{*}}^{1} \frac{1}{y^{2}}\left(\frac{4 \dot{E}_{m}^{2}}{y^{2}}+\frac{\dot{E}_{e q}^{2}}{q_{3}}\right)^{-3 / 2} d y .
$$

Therefore

$$
\frac{\partial A_{\mathrm{sol}}}{\partial p}\left(p^{1}, \dot{\boldsymbol{E}}\right)=\frac{3 q_{3}}{4 \sigma_{0}} \frac{1}{\left(\frac{1}{f_{e}} J_{3}+\dot{E}_{e q}^{2} J_{4}\right)} .
$$

Appendix A.1. Purely deviatoric macroscopic strain-rate: $\dot{E}_{m}=0$.

When the overall strain-rate $\dot{\boldsymbol{E}}$ is purely deviatoric $\left(\dot{E}_{m}=0\right)$, the above quantities of interest read as:

$$
\left.\begin{array}{c}
p^{0}=p^{1}=0, \\
J_{1}=\frac{1-f_{e}}{f_{e}} \dot{E}_{e q}^{-3}, \quad J_{2}=\left(1-f_{e}\right) \dot{E}_{e q}^{-3}, \\
J_{3}=\left(1-f_{b}^{*}\right) \sqrt{q_{3}} \dot{E}_{e q}^{-1}, \quad J_{4}=\frac{1-f_{b}^{*}}{f_{b}^{*}} \dot{E}_{e q}^{-3}\left(q_{3}\right)^{3 / 2}, \\
\frac{\partial A_{\mathrm{sol}}}{\partial p}\left(p^{0}, \dot{\boldsymbol{E}}\right)=\frac{\partial A_{\mathrm{sol}}}{\partial p}\left(p^{1}, \dot{\boldsymbol{E}}\right)=\frac{3 \sqrt{q_{3}}}{4 \sigma_{0}} \frac{1}{1-f_{b}^{*}} \frac{f_{b}^{*} f_{e}}{q_{3} f_{e}+f_{b}^{*}} \dot{E}_{e q} .
\end{array}\right\}
$$

Appendix A.2. Purely hydrostatic macroscopic strain-rate: $\dot{E}_{e q}=0$.

When the overall strain-rate $\dot{\boldsymbol{E}}$ is purely hydrostatic $\left(\dot{E}_{e q}=0\right)$, the above quantities of interest read as:

$$
\begin{gathered}
p^{0}=\frac{2 \sigma_{0}}{3 \sqrt{q_{3}}} \frac{1-f_{b}^{*}}{1-f_{e}} \ln f_{e} \frac{\dot{E}_{m}}{\left|\dot{E}_{m}\right|}, \quad p^{1}=-\frac{2 \sigma_{0}}{3} \ln f_{b}^{*} \frac{\dot{E}_{m}}{\left|\dot{E}_{m}\right|}, \\
J_{1}=\frac{1}{16}\left(1-f_{e}^{2}\right)\left|\dot{E}_{m}\right|^{-3}, \quad J_{2}=\frac{1}{32}\left(1-f_{e}^{4}\right)\left|\dot{E}_{m}\right|^{-3}, \\
J_{3}=\frac{1}{4}\left(1-\left(f_{b}^{*}\right)^{2}\right)\left|\dot{E}_{m}\right|^{-1}, \quad J_{4}=\frac{1}{16}\left(1-\left(f_{b}^{*}\right)^{2}\right)\left|\dot{E}_{m}\right|^{-3}, \\
\frac{\partial A_{\mathrm{sol}}}{\partial p}\left(p^{0}, \dot{\boldsymbol{E}}\right)=\frac{3}{\sqrt{q_{3}} \sigma_{0}} \frac{f_{b}^{*}}{1-f_{b}^{*}} \frac{1}{1+f_{e}}\left|\dot{E}_{m}\right| \\
\frac{\partial A_{\mathrm{sol}}}{\partial p}\left(p^{1}, \dot{\boldsymbol{E}}\right)=\frac{3 q_{3}}{\sigma_{0}} \frac{f_{e}}{1-\left(f_{b}^{*}\right)^{2}}\left|\dot{E}_{m}\right|
\end{gathered}
$$




\section{Appendix B. Properties of $A_{\text {sol }}(\dot{E}, p)$ for ellipsoidal cavities}

Recall that

$$
\sigma_{0}(g+1) \int_{f_{b}^{*}}^{1} \int_{\tilde{f}}^{1} \sqrt{\left.\frac{4 A^{2}}{y^{2}}+\frac{\left(\dot{E}_{m}-A\right)^{2}}{z^{2}} \frac{\tilde{a}^{2}}{q_{3}}+p_{e}, A\right)=}
$$

Proof of Property 3': The optimality conditions of $\tilde{\phi}$ with respect to $A$

$$
\frac{\partial \tilde{\phi}}{\partial A}\left(A_{\mathrm{sol}}, \dot{\boldsymbol{E}}, p\right)=0
$$

Setting:

$$
\tilde{c}^{2}(z)=\frac{1}{4}\left(\tilde{a}^{2}+\tilde{b}^{2} z^{2}\right)
$$

it is readily seen that:

$$
\left.\begin{array}{c}
\frac{\partial \tilde{\phi}}{\partial A}(A, \dot{\boldsymbol{E}}, p)= \\
\sigma_{0}(g+1) \int_{\tilde{f}}^{1} \int_{f_{b}^{*}}^{1} \frac{4 \frac{A}{y^{2}}+\frac{4\left(A-\dot{E}_{m}\right)}{q_{3} z^{2}} \tilde{c}^{2}(z)}{\sqrt{4 \frac{A^{2}}{y^{2}}+\frac{4\left(\dot{E}_{m}-A\right)^{2}}{q_{3} z^{2}} \tilde{c}^{2}(z)+\frac{\dot{E}_{e q}^{2}}{q_{3}}}} d y d z-3\left(1-f_{e}\right) p,
\end{array}\right\}
$$

and therefore the function $p\left(A_{\text {sol }}, \dot{\boldsymbol{E}}\right)$ reads

$$
p\left(A_{\mathrm{sol}}, \dot{\boldsymbol{E}}\right)=\frac{\sigma_{0}(g+1)}{3\left(1-f_{e}\right)} \int_{\tilde{f}}^{1} \int_{f_{b}^{*}}^{1} \frac{4 \frac{A_{\mathrm{sol}}}{y^{2}}+4 \frac{\left(A_{\mathrm{sol}}-\dot{E}_{m}\right)}{q_{3} z^{2}} \tilde{c}^{2}(z)}{\sqrt{4 \frac{A_{\mathrm{sol}}^{2}}{y^{2}}+4 \frac{\left(\dot{E}_{m}-A_{\mathrm{sol}}\right)^{2}}{q_{3} z^{2}} \tilde{c}^{2}(z)+\frac{\dot{E}_{e q}^{2}}{q_{3}}}} d y .
$$

Taking the limit of (B.2) as $A_{\text {sol }} \rightarrow \pm \infty$ yields

$$
\lim _{A \rightarrow \pm \infty} p\left(A_{\mathrm{sol}}, \dot{\boldsymbol{E}}\right)= \pm \tilde{p}^{\infty}, \quad \tilde{p}^{\infty}=\frac{2}{3} \frac{\sigma_{0}(g+1)}{1-f_{e}} \tilde{I}_{0}
$$

where

$$
\tilde{I}_{0}=\int_{\tilde{f}}^{1} \int_{f_{b}^{*}}^{1} \sqrt{\frac{1}{y^{2}}+\frac{\tilde{c}^{2}(z)}{q_{3} z^{2}}} d y d z=\int_{\tilde{f}}^{1} \int_{f_{b}^{*}}^{1} \sqrt{\frac{1}{y^{2}}+\frac{\tilde{a}^{2}+\tilde{b}^{2} z^{2}}{4 q_{3} z^{2}}} d y d z
$$


To prove the more specific result (44), use is made of the optimality condition:

$$
\frac{\partial^{2} \tilde{\phi}}{\partial A^{2}}\left(A_{\mathrm{sol}}, \dot{\boldsymbol{E}}, p\right) \frac{\partial A_{\mathrm{sol}}}{\partial p}=-\frac{\partial^{2} \tilde{\phi}}{\partial A \partial p}\left(A_{\mathrm{sol}}, \dot{\boldsymbol{E}}, p\right)=3\left(1-f_{e}\right)
$$

A straightforward calculation gives:

$$
\frac{\partial^{2} \tilde{\phi}}{\partial A^{2}}(A, \dot{\boldsymbol{E}}, p)=\sigma_{0}(g+1) \int_{\tilde{f}}^{1} \int_{f_{b}^{*}}^{1} \frac{\frac{16 \dot{E}_{m}^{2} \tilde{c}^{2}(z)}{q_{3} y^{2} z^{2}}+\frac{4 \dot{E}_{e q}^{2}}{q_{3}}\left(\frac{1}{y^{2}}+\frac{\tilde{c}^{2}(z)}{q_{3} z^{2}}\right)}{\left.4 \frac{A^{2}}{y^{2}}+4 \frac{\left(\dot{E}_{m}-A\right)^{2} \tilde{c}^{2}(z)}{q_{3} z^{2}}+\frac{\dot{E}_{e q}^{2}}{q_{3}}\right]^{3 / 2}} d y d z .
$$

It follows from (B.6) that as $p$ approaches $\pm \tilde{p}^{\infty}$, i.e. when $A_{\text {sol }}$ approaches $\pm \infty$

$$
\frac{\partial^{2} \tilde{\phi}}{\partial A^{2}}\left(A_{\mathrm{sol}}, \dot{\boldsymbol{E}}, p\right) \sim\left|A_{\mathrm{sol}}\right|^{-3} \frac{\sigma_{0}(g+1)}{2 q_{3}}\left(4 \dot{E}_{m}^{2} \tilde{I}_{1}+\dot{E}_{e q}^{2} \tilde{I}_{2}\right)
$$

where

$$
\tilde{I}_{1}=\int_{\tilde{f}}^{1} \int_{f_{b}^{*}}^{1} \frac{\tilde{c}^{2}(z)}{y^{2} z^{2}}\left(\frac{1}{y^{2}}+\frac{\tilde{c}^{2}(z)}{q_{3} z^{2}}\right)^{-3 / 2} d y d z, \tilde{I}_{2}=\int_{\tilde{f}}^{1} \int_{f_{b}^{*}}^{1}\left(\frac{1}{y^{2}}+\frac{\tilde{c}^{2}(z)}{q_{3} z^{2}}\right)^{-1 / 2} d y d z .
$$

Therefore, according to (B.5):

$$
\left|A_{\mathrm{sol}}\right|^{-3} \frac{\partial A_{\mathrm{sol}}}{\partial p}(\dot{\boldsymbol{E}}, p) \sim \frac{6 q_{3}\left(1-f_{e}\right)}{\sigma_{0}(g+1)} \frac{1}{\left(4 \dot{E}_{m}^{2} \tilde{I}_{1}+\dot{E}_{e q}^{2} \tilde{I}_{2}\right)},
$$

which gives by integration between $p$ and $\tilde{p}^{\infty}$

$$
A_{\text {sol }}(p, \dot{\boldsymbol{E}}) \sim \sqrt{\frac{\sigma_{0}(g+1)}{12 q_{3}\left(1-f_{e}\right)}} \sqrt{4 \dot{E}_{m}^{2} \tilde{I}_{1}+\dot{E}_{e q}^{2} \tilde{I}_{2}} \frac{1}{\sqrt{\tilde{p}^{\infty}-p}} \quad \text { when } \quad p \sim \tilde{p}^{\infty} .
$$

A similar integration can be performed between $-\tilde{p}^{\infty}$ and $p$ to get a similar asymptotic result when $p \sim-\tilde{p}^{\infty}$.

Proof of Property 4': The derivative of $A_{\text {sol }}(p, \dot{\boldsymbol{E}})$ at $p=\tilde{p}^{0}(\dot{\boldsymbol{E}})\left(\right.$ where $A_{\text {sol }}=0$ ) can be obtained from

$$
\frac{\partial^{2} \tilde{\phi}}{\partial A^{2}}\left(0, \dot{\boldsymbol{E}}, \tilde{p}^{0}\right) \frac{\partial A_{\mathrm{sol}}}{\partial p}\left(\tilde{p}^{0}, \dot{\boldsymbol{E}}\right)=-\frac{\partial^{2} \tilde{\phi}}{\partial A \partial p}\left(0, \dot{\boldsymbol{E}}, \tilde{p}^{0}\right)=3\left(1-f_{e}\right) .
$$

It follows from the general expression (B.6) that

$$
\begin{aligned}
\frac{\partial^{2} \tilde{\phi}}{\partial A^{2}}\left(0, \dot{\boldsymbol{E}}, \tilde{p}^{0}\right)= & 4 \sigma_{0}(g+1) \sqrt{q_{3}} \int_{\tilde{f}}^{1} \int_{f_{b}^{*}}^{1} \frac{\frac{4 \dot{E}_{m}^{2} \tilde{c}^{2}(z)}{y^{2} z^{2}}+\dot{E}_{e q}^{2}\left(\frac{1}{y^{2}}+\frac{\tilde{c}^{2}(z)}{q_{3} z^{2}}\right)}{\left[\frac{4 \dot{E}_{m}^{2} \tilde{c}^{2}(z)}{z^{2}}+\dot{E}_{e q}^{2}\right]^{3 / 2}} d y d z \\
& =4 \sigma_{0}(g+1) \sqrt{q_{3}}\left(1-f_{b}^{*}\right)\left[\frac{1}{f_{b}^{*}}\left(4 \dot{E}_{m}^{2} \tilde{J}_{1}+\dot{E}_{e q}^{2} \tilde{J}_{2}\right)+\frac{\dot{E}_{e q}^{2}}{q_{3}} \tilde{J}_{1}\right],
\end{aligned}
$$


where

$$
\tilde{J}_{1}=\int_{\tilde{f}}^{1} \frac{\tilde{c}^{2}(z)}{z^{2}}\left(\frac{4 \dot{E}_{m}^{2} \tilde{c}^{2}(z)}{z^{2}}+\dot{E}_{e q}^{2}\right)^{-3 / 2} d z, \quad \tilde{J}_{2}=\int_{\tilde{f}}^{1}\left(\frac{4 \dot{E}_{m}^{2} \tilde{c}^{2}(z)}{z^{2}}+\dot{E}_{e q}^{2}\right)^{-3 / 2} d z
$$

Therefore

$$
\frac{\partial A_{\mathrm{sol}}}{\partial p}\left(\tilde{p}^{0}, \dot{\boldsymbol{E}}\right)=\frac{3\left(1-f_{e}\right)}{4 \sigma_{0}(g+1) \sqrt{q_{3}}\left(1-f_{b}^{*}\right)} \frac{1}{\left[\frac{1}{f_{b}^{*}}\left(4 \dot{E}_{m}^{2} \tilde{J}_{1}+\dot{E}_{e q}^{2} \tilde{J}_{2}\right)+\frac{\dot{E}_{e q}^{2}}{q_{3}} \tilde{J}_{1}\right]} .
$$

Proof of Property 5': The expression of $\tilde{p}^{1}$ is a direct consequence of (B.2) with

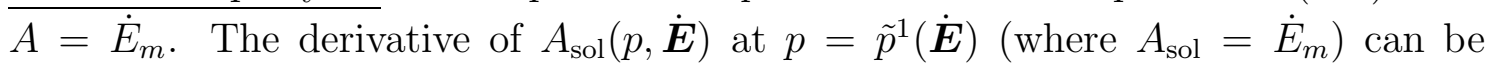
obtained from

$$
\frac{\partial^{2} \tilde{\phi}}{\partial A^{2}}\left(\dot{E}_{m}, \dot{\boldsymbol{E}}, \tilde{p}^{1}\right) \frac{\partial A_{\mathrm{sol}}}{\partial p}\left(\tilde{p}^{1}, \dot{\boldsymbol{E}}\right)=-\frac{\partial^{2} \tilde{\phi}}{\partial A \partial p}\left(\dot{E}_{m}, \dot{\boldsymbol{E}}, \tilde{p}^{1}\right)=3\left(1-f_{e}\right) .
$$

It follows from the general expression (B.6) that

$$
\begin{aligned}
\frac{\partial^{2} \tilde{\phi}}{\partial A^{2}}\left(\dot{E}_{m}, \dot{\boldsymbol{E}}, \tilde{p}^{1}\right) & =4 \frac{\sigma_{0}(g+1)}{q_{3}} \int_{\tilde{f}}^{1} \int_{f_{b}^{*}}^{1} \frac{\frac{4 \dot{E}_{m}^{2} \tilde{c}^{2}(z)}{y^{2} z^{2}}+\dot{E}_{e q}^{2}\left(\frac{1}{y^{2}}+\frac{\tilde{c}^{2}(z)}{q_{3} z^{2}}\right)}{\left(\frac{4 \dot{E}_{m}^{2}}{y^{2}}+\frac{\dot{E}_{e q}^{2}}{q_{3}}\right)^{3 / 2}} d y d z \\
& =4 \frac{\sigma_{0}(g+1)}{q_{3}}(1-\tilde{f})\left[\frac{1}{4}\left(\frac{\tilde{a}^{2}}{\tilde{f}}+\tilde{b}^{2}\right) J_{3}+\dot{E}_{e q}^{2} J_{4}\right],
\end{aligned}
$$

where

$$
J_{3}=\int_{f_{b}^{*}}^{1}\left(\frac{4 \dot{E}_{m}^{2}}{y^{2}}+\frac{\dot{E}_{e q}^{2}}{q_{3}}\right)^{-1 / 2} d y, \quad J_{4}=\int_{f_{b}^{*}}^{1} \frac{1}{y^{2}}\left(\frac{4 \dot{E}_{m}^{2}}{y^{2}}+\frac{\dot{E}_{e q}^{2}}{q_{3}}\right)^{-3 / 2} d y .
$$

Therefore

$$
\frac{\partial A_{\mathrm{sol}}}{\partial p}\left(\tilde{p}^{1}, \dot{\boldsymbol{E}}\right)=\frac{3 q_{3}}{4 \sigma_{0}(g+1)} \frac{1-f_{e}}{1-\tilde{f}} \frac{1}{\left[\frac{1}{4}\left(\frac{\tilde{a}^{2}}{\tilde{f}}+\tilde{b}^{2}\right) J_{3}+\dot{E}_{e q}^{2} J_{4}\right]} .
$$


Appendix B.1. Purely deviatoric macroscopic strain-rate: $\dot{E}_{m}=0$.

When the overall strain-rate $\dot{\boldsymbol{E}}$ is purely deviatoric $\left(\dot{E}_{m}=0\right)$, the above quantities of interest read as:

$$
\left.\begin{array}{c}
\tilde{p}^{0}=\tilde{p}^{1}=0, \\
\tilde{J}_{1}=\frac{1-\tilde{f}}{4}\left(\frac{\tilde{a}^{2}}{\tilde{f}}+\tilde{b}^{2}\right) \dot{E}_{e q}^{-3}, \quad \tilde{J}_{2}=(1-\tilde{f}) \dot{E}_{e q}^{-3}, \\
J_{3}=\left(1-f_{b}^{*}\right) \sqrt{q_{3}} \dot{E}_{e q}^{-1}, \quad J_{4}=\frac{1-f_{b}^{*}}{f_{b}^{*}} \dot{E}_{e q}^{-3}\left(q_{3}\right)^{3 / 2}, \\
\frac{\partial A_{\mathrm{sol}}}{\partial p}\left(\tilde{p}^{0}, \dot{\boldsymbol{E}}\right)=\frac{\partial A_{\mathrm{sol}}}{\partial p}\left(\tilde{p}^{1}, \dot{\boldsymbol{E}}\right)=\frac{3 \sqrt{q_{3}}}{4 \sigma_{0}(g+1)} \frac{1-f_{e}}{1-\tilde{f}} \frac{1}{1-f_{b}^{*}} \frac{f_{b}^{*} \tilde{f}}{q_{3} \tilde{f}+\frac{f_{b}^{*}}{4}\left(\tilde{a}^{2}+\tilde{b}^{2} \tilde{f}\right)} \dot{E}_{e q} \cdot
\end{array}\right\}
$$

Appendix B.2. Purely hydrostatic macroscopic strain-rate: $\dot{E}_{e q}=0$.

When the overall strain-rate $\dot{\boldsymbol{E}}$ is purely hydrostatic $\left(\dot{E}_{e q}=0\right)$, the above quantities of interest read as:

$$
\begin{aligned}
& \tilde{p}^{0}=-\frac{\sigma_{0}(g+1)}{3 \sqrt{q_{3}}} \frac{1-f_{b}^{*}}{1-f_{e}} \frac{\dot{E}_{m}}{\left|\dot{E}_{m}\right|} \tilde{I}_{3}, \\
& \tilde{I}_{3}=\sqrt{\tilde{a}^{2}+\tilde{b}^{2}}-\sqrt{\tilde{a}^{2}+\tilde{b}^{2} \tilde{f}^{2}}+|\tilde{a}| \ln \left(\frac{|\tilde{a}|+\sqrt{\tilde{a}^{2}+\tilde{b}^{2} \tilde{f}^{2}}}{|\tilde{a}| \tilde{f}+\sqrt{\tilde{a}^{2}+\tilde{b}^{2}} \tilde{f}}\right) \text {, } \\
& \tilde{p}^{1}=-\frac{2 \sigma_{0}(g+1)}{3} \frac{1-\tilde{f}}{1-f_{e}} \ln f_{b}^{*} \frac{\dot{E}_{m}}{\left|\dot{E}_{m}\right|}, \\
& \tilde{J}_{1}=\frac{1}{4} \frac{1-\tilde{f}^{2}}{\sqrt{\tilde{a}^{2}+\tilde{b}^{2}}+\sqrt{\tilde{a}^{2}+\tilde{b}^{2} \tilde{f}^{2}}}\left|\dot{E}_{m}\right|^{-3}, \\
& J_{3}=\frac{1}{4}\left(1-\left(f_{b}^{*}\right)^{2}\right)\left|\dot{E}_{m}\right|^{-1}, \quad J_{4}=\frac{1}{16}\left(1-\left(f_{b}^{*}\right)^{2}\right)\left|\dot{E}_{m}\right|^{-3} \text {, } \\
& \frac{\partial A_{\mathrm{sol}}}{\partial p}\left(\tilde{p}^{0}, \dot{\boldsymbol{E}}\right)=\frac{3\left(1-f_{e}\right)}{4 \sqrt{q_{3}} \sigma_{0}(g+1)} \frac{f_{b}^{*}}{1-f_{b}^{*}} \frac{\sqrt{\tilde{a}^{2}+\tilde{b}^{2}}+\sqrt{\tilde{a}^{2}+\tilde{b}^{2} \tilde{f}^{2}}}{1-\tilde{f}^{2}}\left|\dot{E}_{m}\right| \text {, } \\
& \frac{\partial A_{\mathrm{sol}}}{\partial p}\left(\tilde{p}^{1}, \dot{\boldsymbol{E}}\right)=\frac{3 q_{3}}{\sigma_{0}(g+1)} \frac{1-f_{e}}{1-\tilde{f}} \frac{1}{1-\left(f_{b}^{*}\right)^{2}} \frac{4 \tilde{f}}{\tilde{a}^{2}+\tilde{b}^{2} \tilde{f}}\left|\dot{E}_{m}\right| .
\end{aligned}
$$

Remark: For consistency, it can be checked that all results for spherical voids are

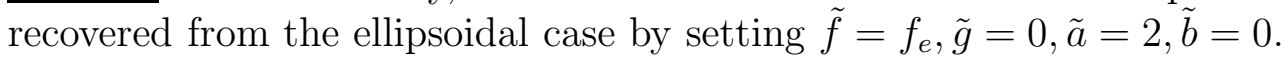

\section{Appendix C. Integrals}

Throughout the paper, use has been made of several integrals which can actually be expressed in closed form. 
Appendix C.1. $I_{1}$ and $I_{2}$

The integrals $I_{1}$ and $I_{2}$ can alternatively be expressed as:

$$
\begin{gathered}
I_{1}=\sqrt{q_{3}}\left(i_{1}+i_{2}-i_{3}-i_{4}\right), \\
I_{2}=\frac{1}{3 \sqrt{q_{3}}}\left[\left(f_{b}^{*}\right)^{2}\left(i_{3}-i_{1}\right)+q_{3}\left(f_{e}^{2}\left(i_{3}-i_{2}\right)+i_{4}-i_{1}\right)+i_{4}-i_{2}\right],
\end{gathered}
$$

where

$$
i_{1}=\sqrt{\left(f_{b}^{*}\right)^{2}+q_{3}}, \quad i_{2}=\sqrt{f_{e}^{2} q_{3}+1}, \quad i_{3}=\sqrt{\left(f_{b}^{*}\right)^{2}+f_{e}^{2} q_{3}}, \quad i_{4}=\sqrt{q_{3}+1}
$$

Appendix C.2. Integrals $I_{0}, \tilde{I}_{0}$, (39) and (55),

The double integrals $I_{0}, \tilde{I}_{0},(39)$ and (55) are special cases of the more general integral

$$
I=\int_{f_{t}}^{1} \int_{f_{b}^{*}}^{1} \sqrt{\frac{P^{2}}{y^{2}}+\frac{Q^{2}}{z^{2}}+R^{2}} d y d z .
$$

Then:

- when $R \neq 0$ :

$$
\begin{aligned}
& I= B_{1}-B_{2}-B_{3}+B_{4}+\frac{P Q}{2 R} \arcsin \left(\frac{L M-N K}{M^{2}+N^{2}}\right) \\
&+ \ln \left[\left(\frac{Q+B_{3}}{f_{t}\left(Q+B_{1}\right)}\right)^{Q}\left(\frac{f_{b}^{*}\left(f_{t} P+B_{3}\right)}{f_{t} P+B_{4}}\right)^{\left(P f_{t}\right)}\right] \\
&+\ln \left[\left(\frac{P+B_{2}}{f_{b}^{*}\left(P+B_{1}\right)}\right)^{P}\left(\frac{f_{t}\left(f_{b}^{*} Q+B_{2}\right)}{f_{b}^{*} Q+B_{4}}\right)^{\left(Q f_{b}^{*}\right)}\right] .
\end{aligned}
$$

where

$$
\begin{gathered}
B_{1}=\sqrt{P^{2}+Q^{2}+R^{2}}, \quad B_{2}=\sqrt{P^{2}+f_{b}^{* 2}\left(Q^{2}+R^{2}\right)}, \\
B_{3}=\sqrt{Q^{2}+f_{t}^{2}\left(P^{2}+R^{2}\right)}, \quad B_{4}=\sqrt{f_{b}^{* 2} Q^{2}+f_{t}^{2}\left(P^{2}+f_{b}^{* 2} R^{2}\right)}, \quad B_{5}=\begin{array}{r}
P Q R, \\
A_{i}=\left(P^{2} Q^{2}-R^{2} B_{i}^{2}\right),
\end{array} \quad C_{i}=2 B_{5} B_{i}, \quad i=1,4, \\
K=A_{1} A_{4}-C_{1} C_{4}, \quad L=A_{1} C_{4}+C_{1} A_{4}, \\
M=A_{2} A_{3}-C_{2} C_{3}, \quad N=A_{2} C_{3}+A_{3} C_{2},
\end{gathered}
$$

- When $R=0$ and $P \neq 0, Q \neq 0$, then

$$
\begin{aligned}
I= & 2\left(B_{1}-B_{2}-B_{3}+B_{4}\right) \\
+ & \ln \left[\left(\frac{Q+B_{3}}{f_{t}\left(Q+B_{1}\right)}\right)^{Q}\left(\frac{f_{b}^{*}\left(f_{t} P+B_{3}\right)}{f_{t} P+B_{4}}\right)^{P f_{t}}\right] \\
+ & \ln \left[\left(\frac{P+B_{2}}{f_{b}^{*}\left(P+B_{1}\right)}\right)^{P}\left(\frac{f_{t}\left(f_{b}^{*} Q+B_{2}\right)}{f_{b}^{*} Q+B_{4}}\right)^{Q f_{b}^{*}}\right],
\end{aligned}
$$


with

$$
\begin{array}{ll}
B_{1}=\sqrt{P^{2}+Q^{2}}, & B_{2}=\sqrt{P^{2}+f_{b}^{* 2} Q^{2}} \\
B_{3}=\sqrt{Q^{2}+f_{t}^{2} P^{2}}, & B_{4}=\sqrt{f_{b}^{* 2} Q^{2}+f_{t}^{2} P^{2}}
\end{array}
$$

- When $R=P=0, Q \neq 0$, then

$$
I=-\left(1-f_{b}^{*}\right)|Q| \ln \left(f_{t}\right)
$$

- When $R=Q=0, P \neq 0$, then

$$
I=-\left(1-f_{t}\right)|P| \ln \left(f_{b}^{*}\right) .
$$

The double integrals $I_{0}, \tilde{I}_{0},(39)$ and (55) can be expressed in closed form:

- The first double integral in (39) $\left(\bar{\Sigma}_{m}^{ \pm}\right)$with $P=2 \bar{A}^{ \pm}, Q=\frac{2\left( \pm 1-\bar{A}^{ \pm}\right)}{\sqrt{q_{3}}}, R=0$, and $f_{t}=f_{e}$

- The second double integral in (39) $\left(\bar{\Sigma}_{e q}\right)$ with $P=2 \bar{A}_{e q}, Q=\frac{2 \bar{A}_{e q}}{\sqrt{q_{3}}}, R=\frac{1}{\sqrt{q_{3}}}$, and $f_{t}=f_{e}$

- $I_{0}$ with $P=1, Q=\sqrt{\frac{1}{q_{3}}}, R=0$, and $f_{t}=f_{e}$.

- $\tilde{I}_{0}$ with $P=1, Q=\frac{\tilde{a}}{2 \sqrt{q_{3}}}, R=\frac{\tilde{b}}{2 \sqrt{q_{3}}}$, and $f_{t}=\tilde{f}$

- The first double integral in $(55)\left(\bar{\Sigma}_{m}^{ \pm}\right)$with $P=2 \tilde{A}^{ \pm}, Q=\frac{\tilde{a}}{\sqrt{q_{3}}}\left( \pm 1-\tilde{A}^{ \pm}\right)$, $R=\frac{\tilde{b}}{\sqrt{q_{3}}}\left( \pm 1-\tilde{A}^{ \pm}\right)$, and $f_{t}=\tilde{f}$.

- The second double integral in $(55)\left(\bar{\Sigma}_{e q}\right)$ with $P=2 \tilde{A}_{e q}, Q=\frac{\tilde{a}}{\sqrt{q_{3}}} \tilde{A}_{e q}, R=$

$$
\sqrt{\frac{1}{q_{3}}+\frac{\tilde{b}^{2}}{q_{3}} \tilde{A}_{e q}^{2}}, \text { and } f_{t}=\tilde{f} .
$$

Appendix C.3. $\tilde{I}_{1}$ and $\tilde{I}_{2}$

$$
\tilde{I}_{1}=4 q_{3}^{3 / 2}\left(\frac{\tilde{i}_{1}-\tilde{i}_{3}}{\tilde{b}^{2}\left(f_{b}^{*}\right)^{2}+4 q_{3}}+\frac{\tilde{i}_{2}-\tilde{i}_{4}}{\tilde{b}^{2}+4 q_{3}}\right)
$$




$$
\begin{gathered}
\tilde{I}_{2}=\frac{4 \sqrt{q_{3}}}{\tilde{b}^{2}}\left[\frac { 1 } { \tilde { i } _ { 5 } } \left[\arctan \left(\tilde{i}_{1} \tilde{i}_{5}\right)+\arctan \left(\tilde{i}_{2} \tilde{i}_{5}\right)-\arctan \left(\tilde{i}_{3} \tilde{i}_{5}\right)\right.\right. \\
\left.\left.-\arctan \left(\tilde{i}_{4} \tilde{i}_{5}\right)\right]-\tilde{i}_{1}-\tilde{i}_{2}+\tilde{i}_{3}+\tilde{i}_{4}\right] \\
\tilde{i}_{1}=\frac{1}{2} \sqrt{\left(f_{b}^{*}\right)^{2}\left(\tilde{a}^{2}+\tilde{b}^{2}\right)+4 q_{3},}, \quad \tilde{i}_{2}=\frac{1}{2} \sqrt{\tilde{a}^{2}+\tilde{f}^{2}\left(\tilde{b}^{2}+4 q_{3}\right)}, \\
\tilde{i}_{3}=\frac{1}{2} \sqrt{\left(f_{b}^{*}\right)^{2}\left(\tilde{a}^{2}+\tilde{b}^{2} \tilde{f}^{2}\right)+4 \tilde{f}^{2} q_{3},} \quad \tilde{i}_{4}=\frac{1}{2} \sqrt{\tilde{a}^{2}+\tilde{b}^{2}+4 q_{3}}, \\
\tilde{i}_{5}=\frac{|\tilde{b}|}{|\tilde{a}| \sqrt{q_{3}}} .
\end{gathered}
$$

\section{Appendix D. Symmetry of the flow surface}

The GTN yield function ((1)) corresponds to a flow surface which is symmetric with respect to the vertical axis passing through the point $\left(\frac{\bar{\Sigma}_{m}^{-}+\bar{\Sigma}_{m}^{+}}{2}, \bar{\Sigma}_{e q}\right)$ in the plane $\left(\Sigma_{m}, \Sigma_{e q}\right)$. The question addressed here is whether this symmetry property is present in the actual macroscopic flow surface (corresponding to the exact minimization in (7) with $\varphi=\varphi^{\text {Gur }}$ over all possible velocity fields), in the flow surface deduced from the upper bound $\Phi^{+}$and in the flow surface deduced from $\bar{\Phi}$.

The exact flow surface cannot even be plotted in the plane $\left(\Sigma_{m}, \Sigma_{e q}\right)$ because of the effect of the third invariant of the stress which complicates the representation of the flow surface even for an isotropic material. In full rigor, the flow surface should be represented in the space of the three invariants of $\boldsymbol{\Sigma}$ (Danas et al. (2008)). Therefore the symmetry of the actual flow surface in the plane of the two first invariants is a ill-posed question and will not be discussed here. It will just be noted in part II of this study that this effect of the third invariant on the overall flow surface is small in the range of porosities of interest to us (less than a few percents).

We discuss here the symmetry of the upper bound corresponding to $\Phi^{+}$. It can be proved in two specific cases, first when $p_{b}=p_{e}=0$ (drained materials see Appendix D.1 for a proof), and second when $f_{b}^{*}=f_{e}$ and $q_{3}=1$ (see Appendix D.2 for a proof). It remains an approximation in general. However, it can be checked that the hydrostatic component (denoted by $\Sigma_{m}^{\text {sol }}$ ) of the stress state on the flow surface where the equivalent stress is maximum is, for all values of the internal pressure $p$, close to the mid point $\frac{\Sigma_{m}^{\text {sol- }}+\Sigma_{m}^{\text {sol }+}}{2}$ between the two hydrostatic points bounding the domain on the hydrostatic axis. As can be seen in Figure D.14, the discrepancy is less than $3 \%$ of the yield stress $\sigma_{0}$.

\section{Appendix D.1. Drained materials $p_{b}=p_{e}=0$}

It follows from the relation (20) that, when $p_{b}=p_{e}=0, \Phi^{+}(16)$ is an even function of $\dot{E}_{m}$ and therefore that its derivative with respect to $\dot{E}_{m}$ is an odd function 

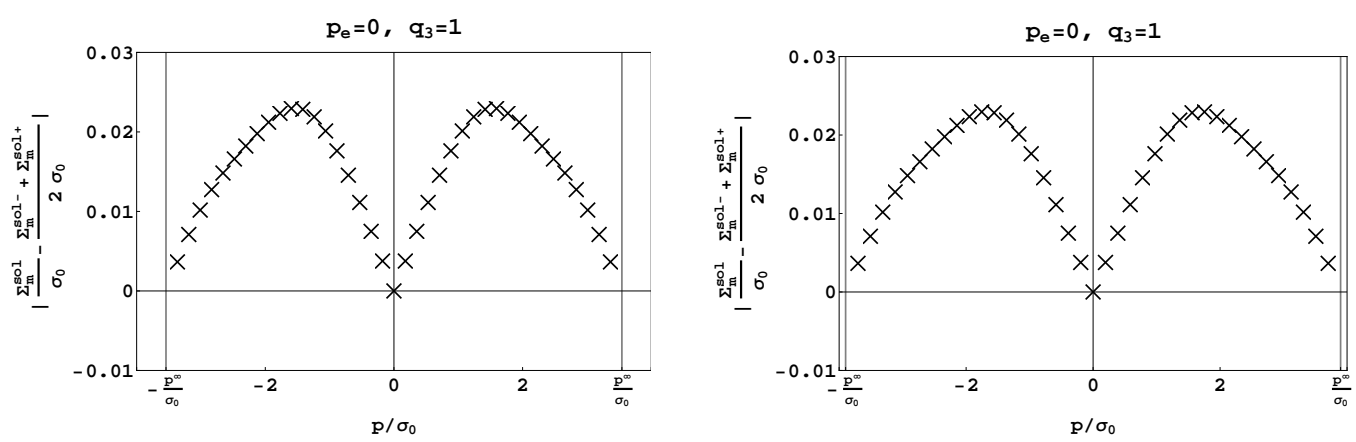

Figure D.14: Spherical voids. Asymmetry of the flow surface obtained with $A_{\text {sol }}$ : (left) $\left(f_{b}, f_{e}\right)=$ $(10 \%, 1 \%)$, (right) $\left(f_{b}, f_{e}\right)=(1 \%, 10 \%)$.

of $\dot{E}_{m}$ whereas its derivative with respect to $\dot{E}_{e q}$ is an even function of $\dot{E}_{m}$ :

$\Sigma_{m}\left(\dot{E}_{m}, E_{e q}\right)=-\Sigma_{m}\left(-\dot{E}_{m}, E_{e q}\right) \quad \Sigma_{e q}\left(\dot{E}_{m}, E_{e q}\right)=\Sigma_{e q}\left(-\dot{E}_{m}, E_{e q}\right), \quad$ when $\quad p_{b}=p_{e}=0$.

This proves the desired symmetry.

Appendix D.2. $f_{b}^{*}=f_{e}$ and $q_{3}=1$

When $f_{b}^{*}=f_{e}$ and $q_{3}=1, A$ and $\dot{E}_{m}-A$ play a symmetric role in the first integral in (17). Therefore it is readily seen that:

$$
\phi(A,-\dot{\boldsymbol{E}}, p)=\phi\left(A+\dot{E}_{m}, \dot{\boldsymbol{E}}, p\right)+3\left(1-f_{e}\right) p \dot{E}_{m} .
$$

Since the minimizer of $\phi$ is unique it follows from relation (D.2) that

$$
A_{\mathrm{sol}}(-\dot{\boldsymbol{E}}, p)=A(\dot{\boldsymbol{E}}, p)+\dot{E}_{m},
$$

and, upon the change of variables $B=A+\dot{E}_{m}$, that the minima are related by

$$
\inf _{A} \phi(A,-\dot{\boldsymbol{E}}, p)=\inf _{B} \phi(B, \dot{\boldsymbol{E}}, p)+3\left(1-f_{e}\right) p \dot{E}_{m},
$$

which implies that the values of the potential $\Phi^{+}$for two opposite strain-rates are related by

$$
\Phi^{+}\left(-\dot{\boldsymbol{E}}, p_{b}, p_{e}\right)=\Phi^{+}\left(\dot{\boldsymbol{E}}, p_{b}, p_{e}\right)+3\left(1-f_{e}\right) p \dot{E}_{m}+6 p_{e} \dot{E}_{m}
$$

Derivating (D.5) with respect to $\dot{\boldsymbol{E}}$ yields

$$
-\frac{\partial \Phi^{+}}{\partial \dot{\boldsymbol{E}}}\left(-\dot{\boldsymbol{E}}, p_{b}, p_{e}\right)=\frac{\partial \Phi^{+}}{\partial \dot{\boldsymbol{E}}}\left(\dot{\boldsymbol{E}}, p_{b}, p_{e}\right)+\left(\left(1-f_{e}\right) p+2 p_{e}\right) \boldsymbol{i}
$$

In other words

$$
\frac{1}{2}\left(\boldsymbol{\Sigma}\left(-\dot{\boldsymbol{E}}, p_{b}, p_{e}\right)+\boldsymbol{\Sigma}\left(\dot{\boldsymbol{E}}, p_{b}, p_{e}\right)\right)=-\left(\frac{\left(1-f_{e}\right)}{2} p+p_{e}\right) \boldsymbol{i}
$$

which shows that the two stresses on the flow surface corresponding to opposite strain-rates are symmetric with respect to the axis $\Sigma_{m}=-\frac{\left(1-f_{e}\right)}{2} p-p_{e}$. 


\section{Appendix E. Hollow ellipsoids}

The inner and the outer boundary of the reference volume are two confocal spheroids (ellipsoids which are rotation invariant around the $z$ axis). Denoting by $a_{1}$ and $b_{1}$ the horizontal and the vertical semi-axes of the inner spheroid (similar notation for the outer spheroid with index 2, see Figure E.15), the void volumefraction and the void aspect-ratio can be expressed as:

$$
f_{e}=\frac{a_{1} b_{1}^{2}}{a_{2} b_{2}^{2}} \text { (volume fraction), } \quad w=\frac{a_{1}}{b_{1}} \quad \text { (aspect ratio). }
$$

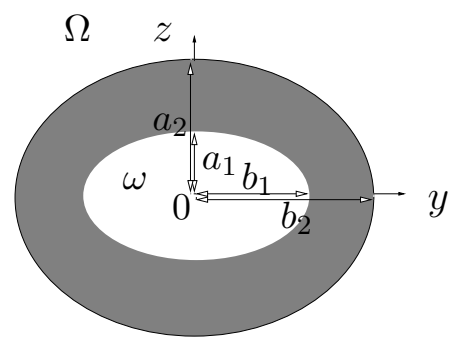

Figure E.15: Notations for the hollow spheroid of reference (Vincent et al., 2009a).

Since the inner and the outer ellipsoids are confocal, the distance $2 c$ between their foci is the same and given by:

$$
c=\sqrt{b_{1}^{2}-a_{1}^{2}}=\sqrt{b_{2}^{2}-a_{2}^{2}} .
$$

A family of confocal ellipsoids with horizontal axis $a$ and vertical axis $b$, parametrized by a scalar $\lambda$, is introduced through the following relations:

$$
a=c \sinh \lambda, \quad b=c \cosh \lambda,
$$

where $\lambda$ varies between $\lambda_{1}$ and $\lambda_{2}$ :

$$
a_{1}=c \sinh \lambda_{1}, \quad b_{1}=c \cosh \lambda_{1}, \quad a_{2}=c \sinh \lambda_{2}, \quad b_{2}=c \cosh \lambda_{2} .
$$

Then, with the following notations:

$$
\begin{gathered}
R(\lambda)=-\frac{a c}{b^{2}}+\operatorname{Arcsin}\left(\frac{c}{b}\right), \quad Z(\lambda)=\frac{2 c}{a}-2 \operatorname{Arcsin}\left(\frac{c}{b}\right), \\
R_{2}=R\left(\lambda_{2}\right), \quad Z_{2}=Z\left(\lambda_{2}\right), \\
|\Omega|=\frac{4}{3} \pi a_{2} b_{2}^{2}, \quad \tilde{\alpha}=\frac{a_{2} b_{2}^{2}}{2 c^{3}}
\end{gathered}
$$

one defines:

$$
J(\lambda)=\frac{4}{3} \frac{\pi b\left(2 a^{2}+b^{2}\right)}{|\Omega|},
$$


and:

$$
\begin{aligned}
Q_{J}(\lambda)= & \frac{4}{9} \frac{b \pi}{|\Omega|}\left\{b^{2}\left[-1+6 R(\lambda) \tilde{\alpha}+3 Z_{2} \tilde{\alpha}\right]^{2} \ldots\right. \\
& +2 a^{2}\left[1-6 Z_{2} \tilde{\alpha}+12 R^{2}(\lambda) \tilde{\alpha}^{2}+12 Z^{2}(\lambda) \tilde{\alpha}^{2}+9 Z_{2}^{2} \tilde{\alpha}^{2} \ldots\right. \\
& \left.\left.+6 Z(\lambda) \tilde{\alpha}\left(1+2 R(\lambda) \tilde{\alpha}-3 Z_{2} \tilde{\alpha}\right)\right]\right\} .
\end{aligned}
$$

The following notations are used for the definition of $\tilde{\phi}$ in the expression (43):

$$
\begin{gathered}
\tilde{f}=\frac{g+f_{e}}{g+1}, \quad g=\frac{4 e_{2}^{3}}{3 \chi \sqrt{1-e_{2}^{2}}}, \quad \chi=\sqrt{\pi^{2}+\frac{32}{3}}, \\
e_{1}=\frac{c}{b_{1}}, \quad e_{2}=\frac{c}{b_{2}}, \quad Z_{2}=\frac{2 e_{2}}{\sqrt{1-e_{2}^{2}}}-2 \arcsin \left(e_{2}\right), \\
\alpha_{G}(e)=-\frac{1-e^{2}}{2 e^{2}}+\frac{\sqrt{1-e^{2}}}{2 e^{3}} \arcsin (e), \quad \alpha_{1}=\alpha_{G}\left(e_{1}\right), \quad \alpha_{2}=\alpha_{G}\left(e_{2}\right), \\
\tilde{a}^{2}=\frac{3}{\kappa^{2}(g+1)^{2}}\left(3-2 \eta+\frac{4 \eta Z_{2}}{\chi g}\right), \quad \tilde{b}^{2}=\left(1-\frac{2}{\chi g} Z_{2}\right)^{2}, \\
\kappa=\left(\frac{2}{3}+\frac{g\left(1-f_{e}\right)\left(g+2 f_{e}+g f_{e}\right)}{3(g+1)^{2}\left(g+f_{e}\right)^{2} \ln \frac{g+1}{g+f_{e}}}\right)^{-1}, \\
\eta=\frac{\kappa\left(1-f_{e}\right)(g+1)\left(g+f_{e}\right) \sinh \left(2 \kappa\left(\alpha_{2}-\alpha_{1}\right)\right)}{(g+1)^{2}+\left(g+f_{e}\right)^{2}+2(g+1)\left(g+f_{e}\right) \tilde{\eta}} \\
\tilde{\eta}=\kappa\left(\alpha_{2}-\alpha_{1}\right) \sinh \left(2 \kappa\left(\alpha_{2}-\alpha_{1}\right)\right)-\cosh \left(2 \kappa\left(\alpha_{2}-\alpha_{1}\right)\right) .
\end{gathered}
$$

